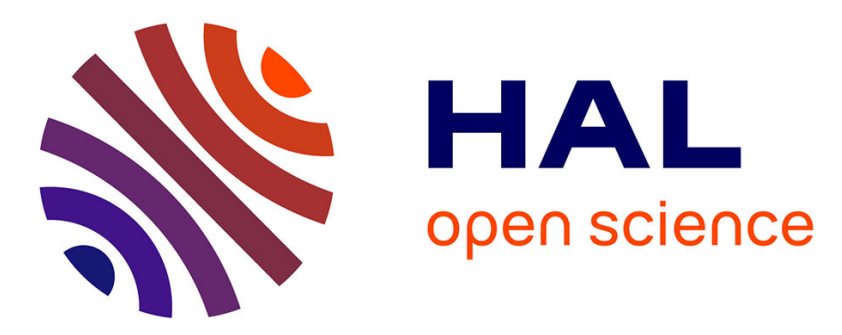

\title{
Numerical investigation of the interaction between laminar to turbulent transition and the wake of an airfoil
}

\author{
A. Ducoin, Jean-Christophe Loiseau, Jean-Christophe Robinet
}

\section{To cite this version:}

A. Ducoin, Jean-Christophe Loiseau, Jean-Christophe Robinet. Numerical investigation of the interaction between laminar to turbulent transition and the wake of an airfoil. European Journal of Mechanics - B/Fluids, 2016, 57, pp.231-248. 10.1016/j.euromechflu.2016.01.005 . hal-01635838

\section{HAL Id: hal-01635838 \\ https://hal.science/hal-01635838}

Submitted on 5 May 2020

HAL is a multi-disciplinary open access archive for the deposit and dissemination of scientific research documents, whether they are published or not. The documents may come from teaching and research institutions in France or abroad, or from public or private research centers.
L'archive ouverte pluridisciplinaire HAL, est destinée au dépôt et à la diffusion de documents scientifiques de niveau recherche, publiés ou non, émanant des établissements d'enseignement et de recherche français ou étrangers, des laboratoires publics ou privés. 


\title{
Numerical investigation of the interaction between laminar to turbulent transition and the wake of an airfoil
}

\author{
A. Ducoin ${ }^{\mathrm{a}, *}$, J.-Ch. Loiseau ${ }^{\mathrm{b}}$, J.-Ch. Robinet ${ }^{\mathrm{c}}$ \\ a LHEEA Laboratory, Ecole Centrale de Nantes (CNRS UMR 6598), 44300 Nantes, France \\ ${ }^{\mathrm{b}}$ Department of Mechanics, Royal Institute of Technology (KTH), SE-100 44 Stockholm, Sweden \\ ${ }^{\mathrm{c}}$ DynFluid Laboratory, ENSAM ParisTech, 75013 Paris, France
}

Keywords:

Transition

Laminar separation bubble

Wing section

Instability

Dynamic Mode Decomposition

\begin{abstract}
A B S T R A C T
The objective of this work is to investigate numerically the different physical mechanisms of the transition to turbulence of a separated boundary-layer flow over an airfoil at low angle of attack. In this study, the spectral elements code NEK5000 is used to simulate the flow over a SD7003 wing section at an angle of attack of $\alpha=4^{\circ}$. Several laminar cases are first studied from $\operatorname{Re}=2000$ to $\operatorname{Re}=10000$, and a gradual increase of the Reynolds number is then performed in order to investigate one transitional case at $R e=20000$. Computations are compared with measurements where the instability mechanisms in the separated zone and near wake zone have been analyzed. The mechanism of transition is investigated, where the DMD (Dynamic Mode Decomposition) is used in order to extract the main physical modes of the flow and to highlight the interaction between the transition and the wake flow. The results suggest that the transition process appears to be physically independent of the wake flow, while the LSB shedding process is locked-in with the von Kármán instability and acts as a sub-harmonic.
\end{abstract}

\section{Introduction}

In both hydrodynamic and aerodynamic communities, the question of transition to turbulence over wing sections has been largely addressed and investigated, mostly because of its crucial role in the design of lifting bodies. In many cases, a good knowledge of the boundary-layer flow regime around the body is required, particularly when modifications of the boundary-layer flow around or on the body occurs, as is the case for transitional flows. A characterization of the transition region combined with a good understanding of the physical process leading to turbulence is then a key in the prediction of the body's performances. It is also of crucial importance for the evaluation of the noise and of the structural vibrations due to flow unsteadiness. In the hydrodynamic field, it has been shown that the control surfaces of Autonomous Underwater Vehicles (AUV) can operate at transitional regimes due to their relatively small scales, with moderate to high Reynolds numbers $\left(5 \cdot 10^{5}<\operatorname{Re}<3 \cdot 10^{6}\right)$. At smaller scale, transition on Micro Air Vehicles has been largely studied because it can take place on more than $50 \%$ of the wing, thus having a direct key role in the flight [1].

\footnotetext{
* Corresponding author.

E-mail address: antoine.ducoin@ec-nantes.fr (A. Ducoin).
}

Transition on lifting bodies is often triggered by a laminar separation and reversed flow due to an adverse pressure gradient. The development of the turbulent flow, which causes a momentum transfer in the wall normal direction, allows the flow to reattach, forming a so called laminar separation bubble (LSB). Downstream of the LSB, the flow is usually highly unsteady and is subject to several complex mechanisms resulting in transition to turbulence.

At first, the reattachment point is subjected to a low frequency oscillation in the shear layer (known as flapping), and is responsible for the first destabilization of the LSB. In the mean time, it has been shown that primary instabilities (Kelvin-Helmholtz vortex and/or Tollmien-Schlichting waves) are responsible for a partial detachment of the LSB yielding to LSB vortex shedding. The physical process leading to the breakdown of coherent structures into smaller scales triggering turbulence is then usually caused by secondary instabilities.

In regard to recent works on laminar-turbulent transition, it appears that some efforts are still to be done in (i) the characterization of the transitional region and its direct effect on pressure distribution hydro/aerodynamic loading (i.e. global effects) and (ii) the understanding of the physical mechanisms that lead to turbulence, including the instability mechanisms and the unsteadiness of the vortex flow that convects downstream of the transitional region and later in the wake. 


\subsection{Previous works of separation induced transition on flat plates}

Many studies have been dedicated to the issue of laminar separation, in particular for aerodynamic applications on flat plates and lifting bodies. The characterization of laminar separation bubbles has been given a special interest, from the pioneering experimental works of Gaster [2] or Tani [3] to recent numerical studies involving direct numerical simulations (DNS). A synthesis of knowledge on the dynamics of LSB can be found in [4].

Simplified geometries where transition is caused by the application of an adverse pressure gradient, such as flat plates [5,6], or by the geometry itself [7], have led to significant improvements in the understanding of the LSB behavior and instability mechanisms. Major reasons are the relative control of laminar separation and the good knowledge of the physical time scales that allow convective perturbations forcing inside the transition region.

The following literature review will concern flat plate with adverse pressure gradient, as it is the closest case to airfoil, and also the most studied.

Direct numerical simulations performed by Alam and Sandham [8] show that complex and multi-scaled structures can exist in the LSB wake. Moreover, the presence of vortex shedding of the bubble has been characterized by Pauley et al. [9]. They found that the frequency of the vortex shedding from the bubble could be made non-dimensional using a Strouhal number $S t_{\theta}$ based on the momentum thickness $\theta_{\text {sep }}$ and the external velocity $\left(v_{\text {ext }}\right)_{\text {sep }}$ at laminar separation.

To understand the physical mechanisms in the transition region, Marxen and Henningson [10] have studied the flow on a flat plate submitted to convective perturbations with large amplitudes. In particular, the authors state that, depending on the length of the bubble (short or long, see Gaster [2]), two distinct behaviors can be observed. The short bubbles are governed by mean flow deformation (MFD), where an algorithmic relationship between the forcing amplitude and the bubble length is observed. For long bubbles, a bursting phenomenon is observed which plays a key role in the turbulent reattachment. Later, the same authors have associated secondary instabilities to be responsible for (i) the deformation of the Kelvin-Helmholtz vortex associated to an elliptic instability in the core of the vortex, and (ii) for the breakdown of the vortex into smaller structures associated with a hyperbolic instability in the braid region between two vortices [6]. Finally, it has been shown that the characteristics of the transitional region highly depend on the flow conditions. Walters and Leylek [11] have demonstrated that under different turbulence intensity levels and inflows, the LSB and transition point can change significantly. In this context, Abu-Ghannam and Shaw [12] have successfully developed an experimental relationship to predict boundary layer transition based on the effect of turbulence, pressure gradient and flow history on flat plates geometries. This experimental correlation is often used to model transition, see [13].

\subsection{Previous works of separation induced transition on wing section}

Depending on the application considered, the physics of the flow on wing geometries can be modified by the curvature of the wing, the angle of attack and by complex dynamics and/or transient effects that are directly taken from operating conditions. Hence, the characteristics of the LSB are directly dependent on its position along the chord. It has been shown both experimentally [14] and numerically [15] that the transitional region appears near the trailing edge for low to moderate angles of attack, and moves toward the leading edge as the angle of attack increases. Moreover, the length of the bubble is inversely related to the adverse pressure gradient. Consequently, the effect of transition may be very different if the laminar separation bubble is located near the leading or trailing edge [16]. It also has to be noted that most studies on lifting bodies investigate natural transition compared to forced transition for flat plates studies [10]. As a consequence, the transition mechanisms and the corresponding coherent structures can be different.

For low angles of attack and/or low Reynolds numbers, the transition region is larger and located closer to the trailing edge, which leads to large effect on aerodynamic performances, a low characteristic frequency of vortex shedding of LSB and strong interactions with the wake flow. An innovative experimental study by Hain et al. [17] uses a time-resolved particle image velocimetry (TR-PIV) system to determine the dominant frequencies of the transition process and of the flapping of the bubble along a SD7003 airfoil at $R e=66000$. The authors identify dominant structures which lead to large magnitudes in the frequency spectrum. The same physics on the SD7003 geometry was investigated for Reynolds numbers between $20000<\operatorname{Re}<66000$, and for different angles of attack using experimental [18-21] and numerical methods [22,23]. At $R e=20000$, transition is observed near the trailing edge and a vortex associated with a Kelvin-Helmholtz instability is convected into the foil's wake at a characteristic frequency $0.0095 \leq S t_{\theta} \leq$ 0.011 [18]. However, the vortical flow in the wake has not been investigated. For $R e=66000$, transition is stronger and a burst phenomenon is associated with a fast transition to turbulence [18,22] even if Kelvin-Helmholtz instabilities are still observed. Recently, Jones et al. [24] performed a numerical analysis of a LSB in the case of an airfoil at $\alpha=5^{\circ}$ and a low Reynolds number $R e_{c}=50000$. The authors investigated the instability mechanisms leading to the breakdown to turbulence. As Marxen and Henningson [10] stated for the case of a flat plate, this breakdown is linked to the combination of two absolute instabilities: an elliptic instability and a mode$B$ or hyperbolic instability. An important conclusion was that, in the absence of convectively driven transition within the shear layer, an absolute instability can take place and makes the influence of free stream turbulence weaker.

At high angles of attack and/or high Reynolds numbers, transition is located at the leading edge and is characterized by a short laminar separation bubble. Transition occurs brutally in a very high pressure gradient region and its behavior is characterized by high frequency oscillations of the laminar separation bubble shedding [16]. Moreover, it has been shown that at higher angles of attack, the laminar separation was not able to reattach, and that the bubble may help the destabilization of the boundarylayer flow at the leading edge potentially causing stall to occur, as shown numerically by Shelton et al. [25] and Genç [26]. Genç et al. [14] also showed that as the Reynolds number increases, the stall characteristic is strongly modified and the mild stall occurs whereas the abrupt stall occurs at lower Reynolds numbers. They also highlighted that the stall angle decreases with decreasing Reynolds number, which induces the short bubble burst at higher angles of attack, and causes long bubble to occur. This has been validated by the computations of Karasu et al. [15], who show the importance to take into account for the transition in the numerical model

\subsection{Objective and structure of the paper}

Studies on airfoils show a transition by Laminar Separation Bubbles for which mechanisms associated with flapping of the shear layer and primary instability have been demonstrated. It appears however that further comparisons with flat plate studies are still necessary and could bring new elements in the understanding of the physics of transition. On the one hand, investigations on flat plate geometries have indeed led to detailed analysis of the instability mechanisms responsible for transition to turbulence. On the 
a

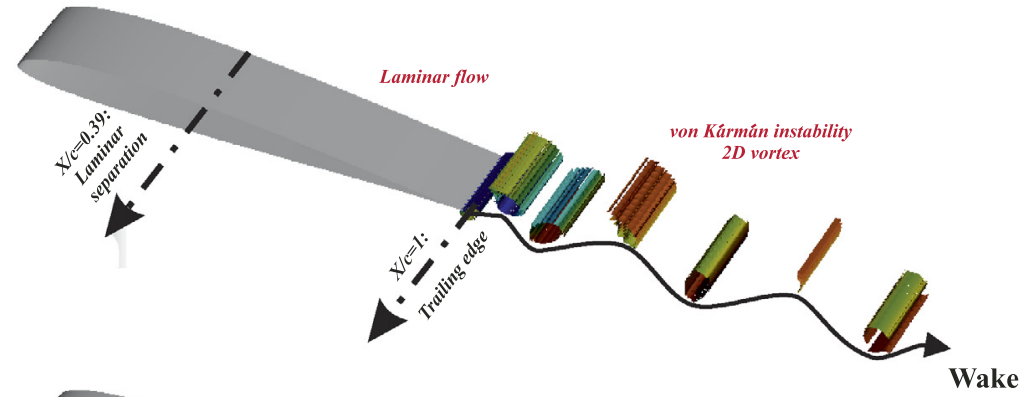

b
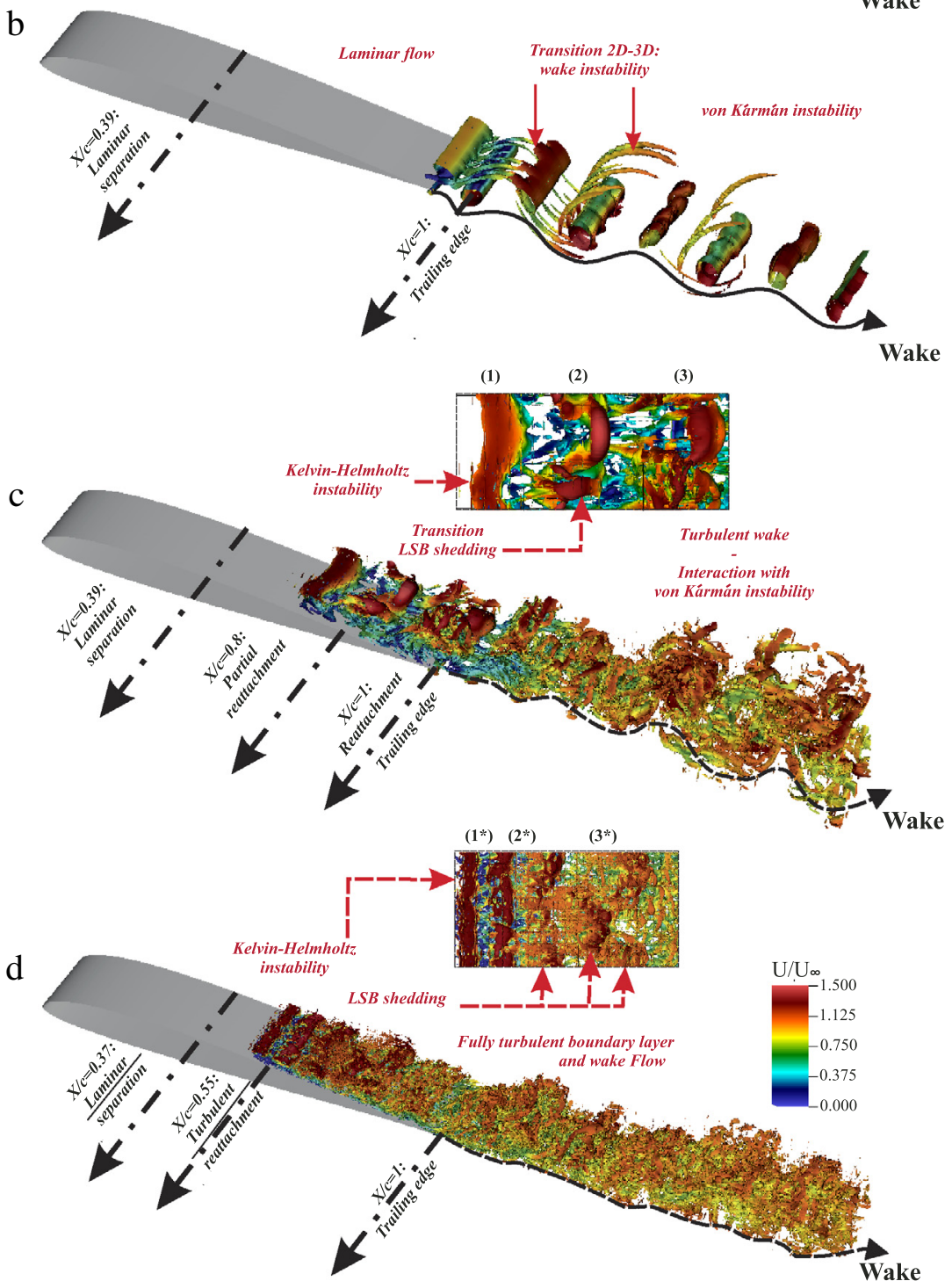

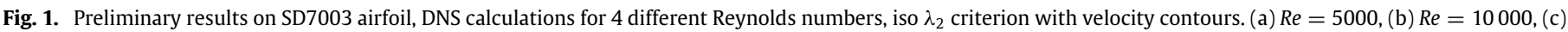
$R e=20000,(\mathrm{~d}) \operatorname{Re}=60000$.

other hand, the wing sections studies have shown similarities with flat plate studies without clearly identifying these mechanisms. If the origin of the transition and the vortex physics should be globally the same, the way Kelvin-Helmholtz vortices are convected and the progressive breakdown to turbulence could be different due to the wing geometry. In particular, the Kelvin-Helmholtz instability can strongly interact with a von Kármán vortex in the case where vortical structures of the transition stay coherent while being convected down to the trailing edge. This can also have an effect on the wake flow, which can in turn modify the transition region. Moreover, the presence of known coherent structures in the wake of the LSB that have also been identified in flat plate studies bring us to consider secondary instabilities in the transition process.

The flow fields past the SD7003 airfoil at an angle of attack of $\alpha=4^{\circ}$ are depicted in Fig. 1 for different Reynolds numbers. These figures highlight that, after the onset of a two-dimensional von Kármán instability ( $R e=5000)$, a wake instability appears at $R e=$ 10000 and is responsible for a three-dimensionalization of the wake flow. The flow around the airfoil starts to transition around $R e=20000$ near the trailing edge, where Kelvin-Helmholtz instability occurs (1), and is then shed (2) downstream the LSB. The boundary-layer flow is however not yet fully turbulent and 


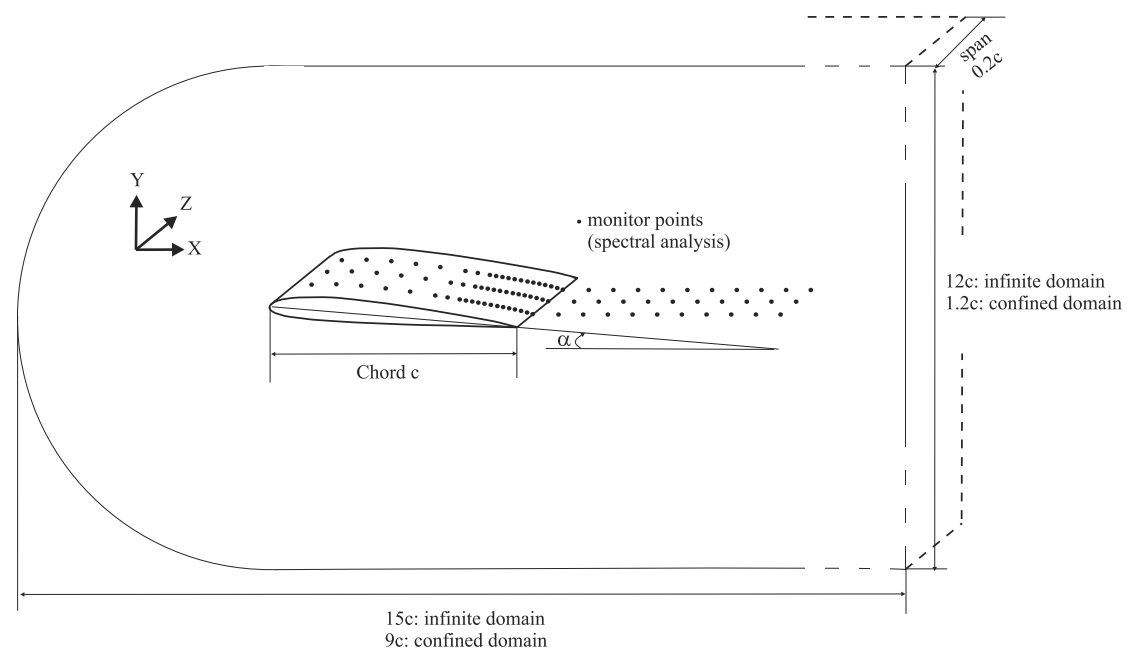

Fig. 2. Computational domain of the SD7003 wing.

vortical structures are still coherent at the trailing edge (3) such that the von Kármán instability still occurs. The higher Reynolds number case ( $R e=60000$ ) has a more classical transition process where the transition occurs around the mid-chord of the airfoil with Kelvin-Helmholtz instability $\left(\left(1^{*}\right)\right.$ and $\left.\left(2^{*}\right)\right)$ followed by LSB shedding (3). In this case, the boundary-layer becomes fully turbulent downstream the LSB.

Whereas this last case has been widely investigated both experimentally and numerically, the lower Reynolds number $R e=20000$ case has received much less attention. In particular, the interaction between the Kelvin-Helmholtz and von Kármán primary instabilities has not been studied because the wake is usually not captured though it can strongly influence the flow behavior and the transition mechanisms. As a consequence, the $R e=20000$ case is investigated herein with particular attention being paid to the coupling mechanisms between the two primary instabilities.

DNS computations are performed with the open-source spectral elements code NeK5000 [27] on a SD7003 geometry at an angle of attack $\alpha=4^{\circ}$. The main objectives of the present study are (i) to investigate the interaction between the laminar-turbulent transition and its interaction with the wake flow characterized by von Kármán instability, and (ii) to present a new approach of investigation that could bring a better knowledge of the instabilities responsible for the unsteadiness. After a presentation of the numerical methods, a mesh sensitivity analysis is presented at a laminar Reynolds number $R e=5000$ for an infinite, 2D domain, and the effect of confinement is briefly analyzed for $R e=5000$ and $R e=10000$. Three-dimensional computations for a transitional Reynolds number of $R e=20000$ in a confined test section are then exposed and compared with PIV experiments of Burgmann et al. [18] which lead to a characterization of the LSB region. The unsteady region downstream of the LSB is finally investigated, including an analysis of the vortex flow and a time-space investigation using spectral analysis and Dynamic Mode Decomposition (DMD).

\section{Problem statement}

The dynamics of a three-dimensional incompressible flow of a Newtonian fluid are described by the Navier-Stokes equations

$$
\begin{aligned}
\dot{\mathbf{U}} & =-(\mathbf{U} \cdot \nabla) \mathbf{U}-\nabla P+R e^{-1} \Delta \mathbf{U} \\
\nabla \cdot \mathbf{U} & =0
\end{aligned}
$$

where $\mathbf{U}=\left(U_{x}, U_{y}, U_{z}\right)^{T}$ is the velocity vector and $P$ the pressure term. A sketch of the geometry considered is depicted in Fig. 2. The wing has a SD7003 section and is made non-dimensional using the chord length $c$. The velocity is non-dimensionalized by the upstream velocity $U_{\infty}$ and the flow conditions are set according to the Reynolds number $R e=U_{\infty} c / v$, where $v$ is the kinematic viscosity of the considered fluid.

The Navier-Stokes equations are solved using the flow solver NEK5000 developed at Argonne National Laboratory by Fischer et al. [28]. It is based on the spectral elements method (SEM), introduced by Patera [29], which provides spectral accuracy in space while allowing for the geometrical flexibility of finite element methods. Spatial discretization is obtained by decomposing the physical domain into spectral elements within which the velocity is defined on Gauss-Lobatto-Legendre (GLL) nodes and the pressure field on Gauss-Legendre (GL) nodes. The solution to the Navier-Stokes equations is then approximated within each element as a sum of Lagrange interpolants defined by an orthogonal basis of Legendre polynomials up to degree $N$. Depending on the Reynolds number considered, the results presented in this paper have been obtained with a polynomial order between $N=4$ and $N=6$. The number of spectral elements in the mesh ranges from 6000 for $R e=5000$ up to 153000 for $R e=20000$. The convective terms are advanced in time using an extrapolation of order 3 , whereas the viscous terms use a backward differentiation of order 3 as well, resulting in the time-advancement scheme labeled BDF3/EXT3. NEK5000 employs the MPI standard for parallelism [30,31] and parallel computations have been performed on up to 1024 processors. For further details about the spectral elements method, the reader is referred to the books by Deville et al. [32] and Karniadakis and Sherwin [33].

\section{Numerical setup and convergence study}

Two computational domains considered are shown in Fig. 2. A non-confined two-dimensional C-type mesh, having a total height of $12 c$ and a length of $15 c$, is first used for the validation of laminar cases $(5000<R e<10000)$. A similar three-dimensional C-type mesh with confinement is used for the transitional case $(R e=20000)$. It is characterized by a tunnel section of $1.2 \mathrm{c}$ height representing a blocking effect of $10 \%$ at an angle of attack $\alpha=4^{\circ}$ and a domain length of $9 c$. The span has been reduced to $0.2 c$ to reduce computational cost. As observed numerically by Uranga et al. [23], this span is sufficient to take into account the three-dimensional effects. This also corresponds to the volume of measurements of Burgmann et al. [18], Zhang et al. [19] and Olson et al. [20] used to capture at least one vortex structure in the spanwise direction for $R e=20000$. This aspect has been 
Table 1

Parameters for the six different meshes.

\begin{tabular}{lclrrrr}
\hline Mesh type (2D,3D) & $N_{\text {wing }}$ & $N_{\text {normal }}$ & $N_{\text {wake }}$ & $N_{e}$ & Order & Degrees of freedom \\
\hline 2D & 50 & 30 & 50 & 6400 & $4-6$ & $102400-230400$ \\
2D & 70 & 40 & 100 & 11500 & $4-6$ & $184000-414000$ \\
3D & 100 & 50 & 130 & 153000 & $4-6$ & $9792000-33048000$ \\
\hline
\end{tabular}

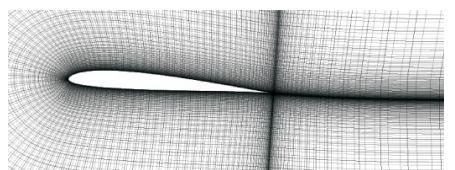

(a) 2D-infinite, $N_{e}=6400$, 4th Order

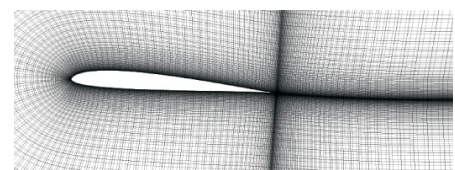

(b) 2D-infinite, $N_{e}=11500,4$ th Order.

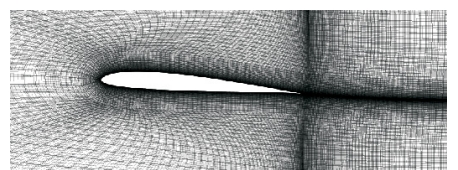

(c) 2D-infinite, $N_{e}=15300 \times 10,4$ th Order.

Fig. 3. Examples of three different meshes in the $(x, y)$ plan.

investigated by carrying out a DNS with a larger span, which is shown at the end of the convergence study.

A velocity condition is set at the inlet, and $\nabla \mathbf{U} \cdot \mathbf{x}=0$ is set at the outlet. In order to reduce the CPU time, symmetries are imposed on the horizontal walls, whereas a no slip condition is set on the wing surface. For the 3D computations, periodic boundary conditions are imposed on the vertical side planes of the domain. Unlike an attached boundary-layer, where the transition position heavily depends on the environmental forcing, in a separated boundarylayer and for the range of Reynolds number studied, the transition position is generally in the separated zone and less dependent on how it is forced. Furthermore, in experiments [18] the transition is natural. Hence, we have chosen not to introduce any forcing.

Three sets of 30 monitor points are used to record the pressure and velocities along the chord and in the wake in order to lead a detailed spectral analysis. As shown in Fig. 2, it is refined in the transitional region while it has a constant spacing in the wake and near the leading edge. Mesh refinement is also used in the vicinity of the leading edge, the trailing edge and in the close wake in order to correctly capture the vortical flow.

Table 1 summarizes the different meshes tested. The mesh is modified according to the number of elements $N_{\text {wing }}$ along the chord, the distance between the wing and the horizontal walls $\left(N_{\text {normal }}\right)$ and the wake $\left(N_{\text {wake }}\right)$. The finer two-dimensional mesh for the laminar cases (see convergence study next section) is constructed using 70 spectral elements along the suction and pressure sides of the wing, 100 elements in the wake and 45 elements are set between the wing and the horizontal walls. The three-dimensional mesh used for the transitional cases is significantly finer, with 100 elements being used to discretize the suction and pressure sides and 130 elements in the wake. 10 spectral elements are set in the spanwise direction $z$ leading to $\Delta x / \Delta z \approx 1$ along $80 \%$ of the wing surface. The boundary-layer mesh is kept constant and has 25 elements in the wall-normal direction. The first cell size has been set in order to obtain $y_{+}=$ $y u_{\tau} / \nu \approx 1$ for a partially turbulent case $(\operatorname{Re}=60000)$, since the boundary-layer is mostly laminar in the cases investigated in this paper. The main mesh parameters for the DNS calculation at $R e=20000$ are $y_{+}<1, x_{+} \approx z_{+} \approx 3$.

Based on this setup, a mesh sensitivity analysis is performed. The influence of the number of elements and of the order of Legendre polynomials used is investigated for a laminar Reynolds number $R e=5000$. As the transitional Reynolds number is validated using a confined fluid domain, this effect is also analyzed. The objective is to set a mesh that is able to simulate accurately the flow at the lower Reynolds number $(R e=5000$ with 4th order polynomials), which can be used as an input of the transitional Reynolds number case where the polynomial order is increased accordingly (6th order). Fig. 3 shows an example of three meshes using 4 th order Legendre polynomials. The first two meshes are set with an unconstrained domain in order to validate the DNS code on a wing section at $R e=10000$ against the LES computations of Uranga et al. [23]. Once this mesh is validated, a finer mesh with confinement is tested that matches the tunnel test section used in [18] for the $R e=20000$ case.

Fig. 4 depicts the velocity field for the different meshes reported in Table 1. The flow is quasi-stationary around the wing and detaches approximately at a distance of $0.35 c$ from the leading edge in all cases. von Kármán vortices are observed in the wake. As shown in Fig. 4(a), these vortices are not correctly captured by the coarser mesh. This figure also demonstrates that an increase of the order of the Legendre polynomials results in a better prediction of the vortex flow as highlighted in Fig. 4(b) and (c). The confined mesh gives a very accurate prediction of the velocity field, with a slight reduction of the wave length of the von Kármán vortex.

The sensitivity of the displacement and momentum thicknesses with respect to the different meshes considered is shown in Fig. 5. In addition, results obtained with the potential flow code XFoIL [34] are also presented. The boundary-layer characteristics are accurately predicted for all the meshes up to $X / c=0.7$, where the wrong prediction of the wake observed in Fig. 4 for the low order leads to errors near the foil trailing edge.

To ensure that the reduced span does not force the spanwise wavelength of the vortices in the transitional case ( $R e=20000)$, a simulation with a larger span $(0.4 c)$ has been carried out. The vortical structures, identified using the $\lambda_{2}$ criterion, are shown in Fig. 6. The two calculations predict quite the same physics in the transition region. The wavelength of the Kelvin-Helmholtz vortex is approximately $0.18 c$ for the larger span calculation, and around $0.2 c$ for the reduced span calculation. The larger difference observed is the streamwise amplitude, which seems to be amplified by the span reduction. Despite these differences, we can observe that it leads to an equivalent vortex shedding and hence to the same transition mechanisms.

\section{Results}

\subsection{Validation on a laminar case, $R e=10000$}

\subsubsection{Comparison with Uranga et al. [23]}

As a first step in our investigations, the numerical procedure is first validated on a laminar case ( $R e=10000)$ already investigated by Uranga et al. [23] using a LES approach and a finite elements discretization. The two-dimensional mesh considered by the former authors is made of 4400 finite elements of order 3 resulting in 39600 degrees of freedom. As a comparison, the present two-dimensional mesh is made of 11500 spectral elements with Lagrange polynomials of order 6 , resulting in 414000 degrees of freedom. Based on the value of $x_{+}, y_{+}$and 


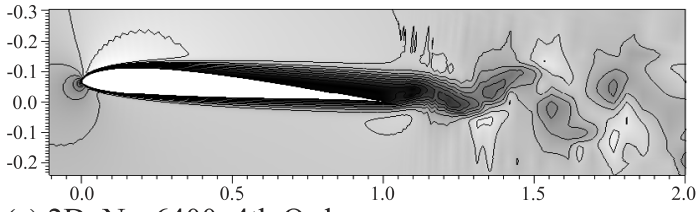

(a) $2 \mathrm{D}, \mathrm{Ne}=6400$, 4 th Order

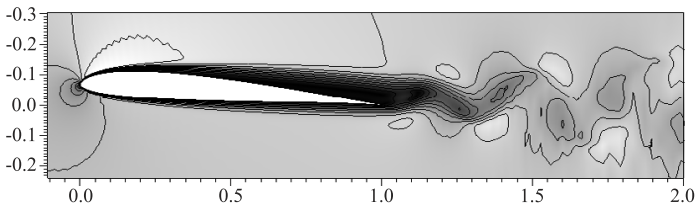

(c) $2 \mathrm{D}, \mathrm{Ne}_{\mathrm{e}}=11500,4$ th Order

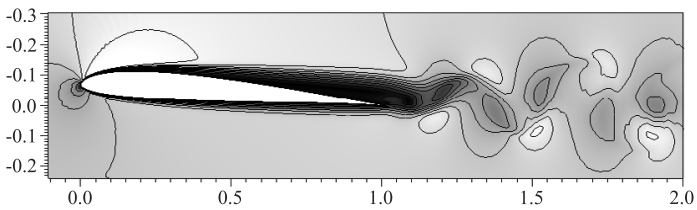

(e) $3 \mathrm{D}, \mathrm{Ne}=15300 \times 10,4$ th Order

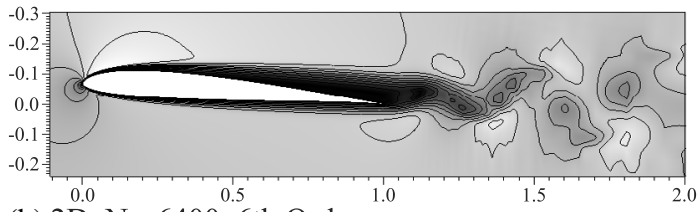

(b) $2 \mathrm{D}, \mathrm{Ne}_{\mathrm{e}}=6400,6$ th Order

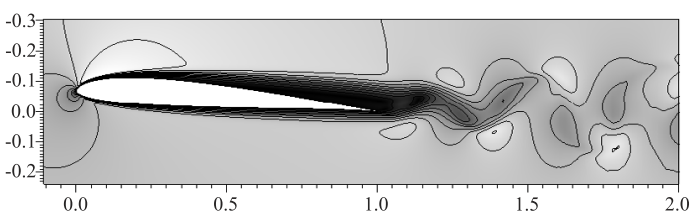

(d) $2 \mathrm{D}, \mathrm{Ne}_{\mathrm{e}}=11500,6$ th Order

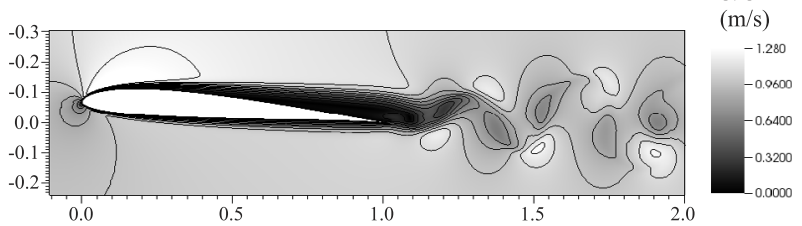

(f) $3 \mathrm{D}, \mathrm{Ne}_{\mathrm{e}}=15300 \times 10,6$ th Order

Fig. 4. Mesh convergence study: effect of number of elements and of the confinement on the velocity field at $R e=5000$.

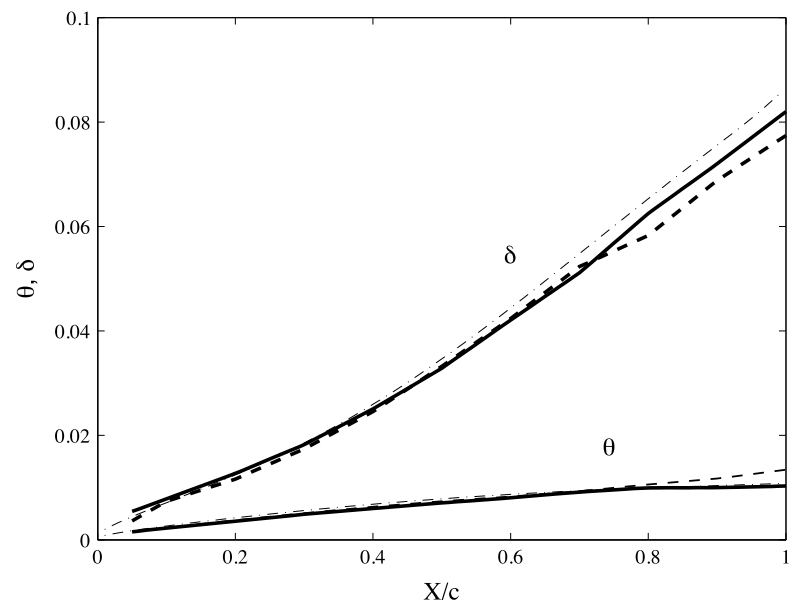

Fig. 5. Mesh convergence study: effect of number of elements and of the confinement on boundary-layer characteristics. (-) fine mesh, order 6, (- - -) coarse mesh, order 4, (- --) Xforl at a Reynolds number $R e=5000$.

$z_{+}$given in Section 3, the present calculations are clearly in the range of a direct numerical simulation compared to the work of Uranga et al. [23]. The instantaneous and time-averaged spanwise non-dimensional vorticity fields $\varpi_{z}=\varpi_{z}^{\star} c / U_{\infty}$ are depicted in Fig. 7(a) and (c), respectively, and compared with those of Uranga et al. [23]. As shown by these figures, a very good agreement is obtained between the two computations. It has to be noted however that, due to the finer mesh used in the present work, the von Kármán vortices, which shed from the trailing edge of the airfoil are captured more accurately than in [23], see Fig. 7(a) and (b). Comparison of the boundary-layer characteristics and of the friction coefficient are shown in Fig. 8. Once again, a very good agreement is observed between the current DNS and the LES of Uranga et al. [23], in particular the non-linearities near the trailing edge due to boundary-layer unsteadiness, while the potential code XFoIL becomes less accurate because of boundarylayer separation.

\subsubsection{Influence of the vertical confinement}

While the previous validation considered an infinite twodimensional domain, the investigation of the transition at $R e=$

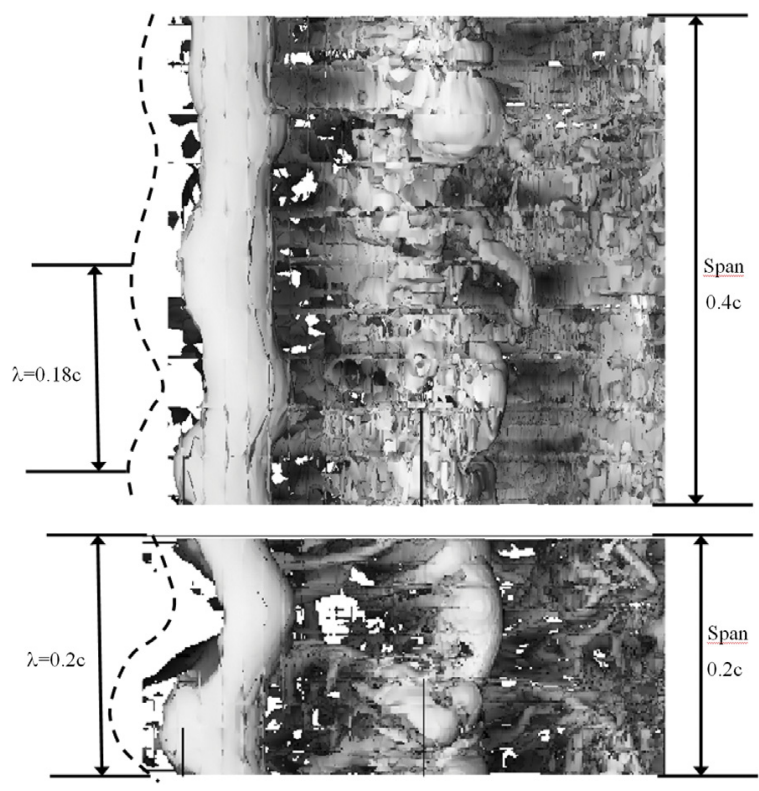

Fig. 6. Influence of span on the spanwise wave length of Kelvin-Helmholtz vortex top view between $0.7 c$ and $1.2 c, R e=20000$.

20000 is performed using a three-dimensional confined domain. Hence, in order to identify the effect of confinement, a comparison between the flow at $R e=10000$ in the two-dimensional infinite domain and the one in the three-dimensional confined domain, similar to the experimental one used by Burgmann and Schröder [35], is presented. Fig. 9(a) shows the time-evolution of the vertical velocity recorded by a probe located at $X / c=$ 0.8 in both domains, where $t=t^{\star} U_{\infty} / c$, while Fig. 9(b) depicts the corresponding Discrete Fourier Transform (DFT). In both cases, the Fourier spectrum exhibits a peak at $S t_{d} \simeq$ 0.24 , where $S t_{d}=f d / U_{\infty}$ is a Strouhal number based on the airfoil thickness $d$. The confinement in the three-dimensional case however causes a slight shift of this characteristic Strouhal number toward higher frequencies. Additional high frequency peaks can moreover be observed compared to the two-dimensional infinite domain. 

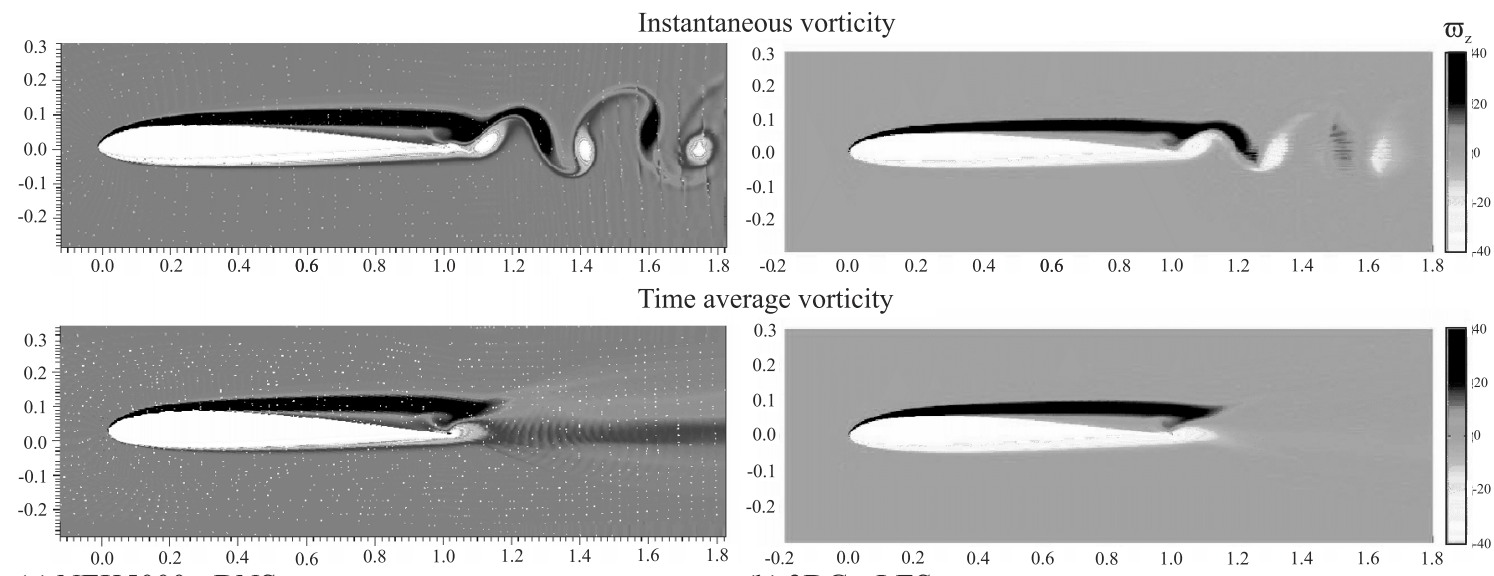

(a) NEK5000 - DNS

(b) $3 \mathrm{DG}$ - LES

Fig. 7. Comparison of the mean and instantaneous vorticity fields, (a) present computation with NeK5000, (b) LES computation of Uranga et al. [23] at Re $=10000$ in the infinite domain.
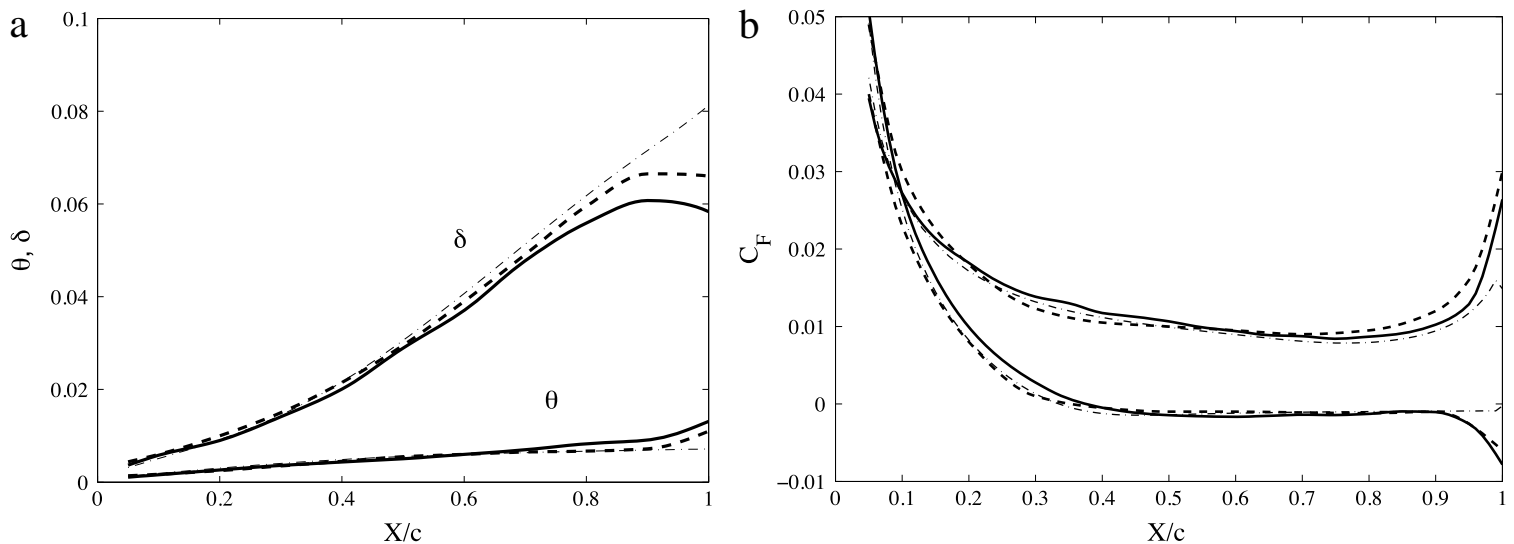

Fig. 8. Comparison of boundary-layer characteristics (a) and skin friction coefficient (b), (-) present DNS, (- - -) LES from Uranga et al. [23], (- .-) XFolL at Re = 10000 in the infinite domain.
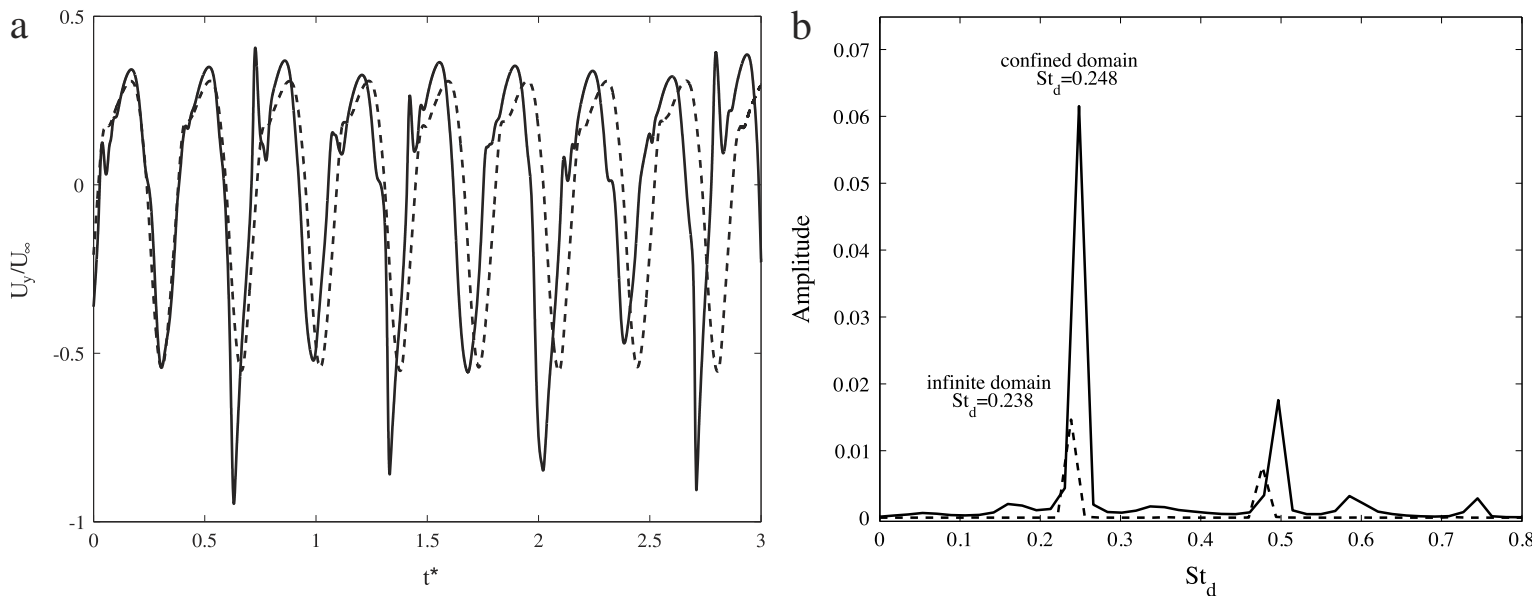

Fig. 9. Comparison of (a) local velocity and (b) spectrum of local velocity at $X / c=0.8$ for a $2 \mathrm{D}$ infinite domain (-) and a $3 \mathrm{D}$ confined domain (- - ) at $R e=10000$.

\subsubsection{Dynamic Mode Decomposition}

The present Dynamic Mode Decomposition (DMD) aims at illustrating and validating DMD on a simple case where the physics are known, as well as to characterize the wake flow. This analysis is based on a sequence of 200 snapshots of the velocity field sampled at a constant sampling period $\Delta t=0.03$ such that each period of the von Kármán vortex shedding is discretized using 12 snapshots. A comprehensive description of the algorithm used is given in Appendix. Fig. 10(a) depicts the eigenspectrum of the Koopman operator, while Fig. 10(b) shows the amplitude of each mode with respect to the associated frequency. As can be seen, the dynamics are dominated by a single frequency $S t_{d}=$ 0.238 , already identified from the spectral analysis in the previous section. Its first three harmonics are also visible in this spectrum. Fig. 11 depicts the spatial distribution of the leading Koopman mode $\left(S t_{d}=0.238\right)$ and of its first harmonic $\left(S t_{d}=0.476\right)$. 

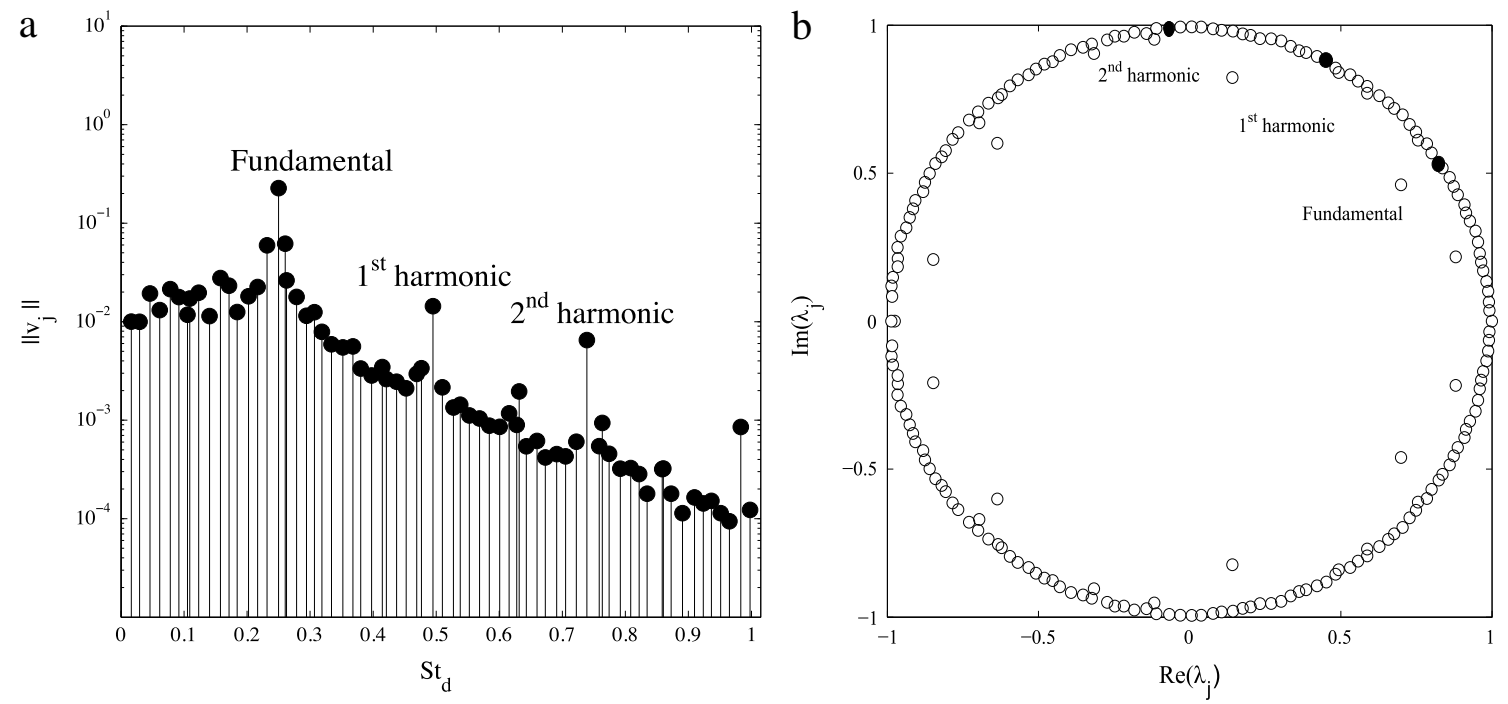

Fig. 10. Dynamic Mode Decomposition, (a) Koopman spectrum, (b) eigenvalues along the unit circle, $R e=10000$.
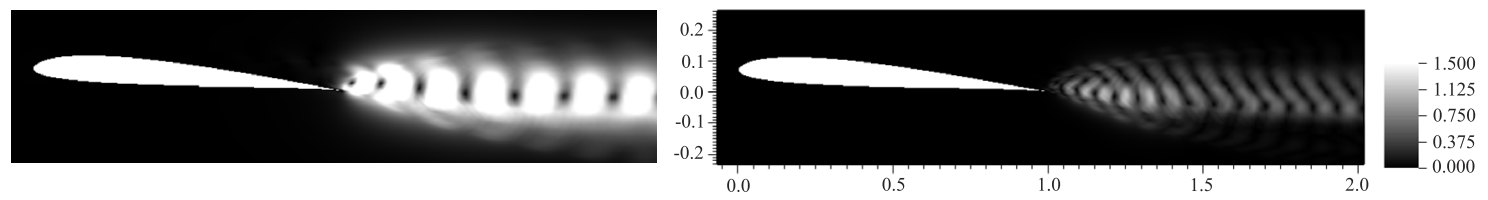

Fig. 11. Velocity field $\|U\|$ for the two leading Koopman modes in the $(X, Y)$ plane at $R e=10000$.

The velocity field of the leading Koopman mode is clearly related to a von Kármán vortex street with a streamwise wavelength of about $0.125 c$. On the other hand, its first harmonic is characterized by lower amplitude oscillations and a streamwise wavelength of about $0.0625 c$, i.e. twice as small as the fundamental one. Finally, these spatial distributions show that this von Kármán vortex shedding is solely related to what occurs in the vicinity of the trailing edge and in the wake, thus highlighting that the laminar separation bubble plays no direct role in the present low Reynolds number dynamics.

\subsection{From the laminar to the transitional flow}

\subsubsection{Influence of the Reynolds number}

Computations have been performed in a three-dimensional confined domain (similar to the experimental one of Burgmann and Schröder [35]) for Reynolds numbers ranging from $R e=5000$ (laminar) up to $R e=20000$ (transitional). The effect of an increase of the Reynolds number is shown in Fig. 12. It depicts the spanwise non-dimensional vorticity field, both in a vertical and spanwise plane, for various Reynolds numbers. As shown by Fig. 12(a), at $R e=5000$, the flow exhibits a laminar and two-dimensional von Kármán vortex shedding. Increasing the Reynolds number to $R e=10000$ (see Fig. 12(b)) does not qualitatively change the flow. A spanwise modulation of the von Kármán vortices can however be observed. This spanwise modulation might result from a secondary instability of the vortices similarly to what has been observed in the wake of an infinite two-dimensional cylinder [36]. Finally, the flow at $R e=20000$ is very different from the lower Reynolds number ones, as shown in Fig. 12(c). A strong three-dimensionalization of the flow in the wake can be observed. Moreover, while for Re = 5000 and 10000 unsteadiness is only observed in the wake, for $R e=20000$ it can be observed that vortices seem to be shed already from $X / c \simeq 0.7$. The resulting complex multi-scaled flow then results from the transition taking place in the vicinity of the trailing edge and to the progressive breakdown to turbulence in the wake. The following investigation will focus on this $R e=20000$ case and aims at a better characterization of the flow as well as at shedding some light on the different physical mechanisms responsible for the laminar-turbulent transition taking place.

\subsubsection{Global characterization of the flow at $R e=20000$}

The mean flow field, along with streamlines in the vicinity of the trailing edge, is depicted in Fig. 13(a), while Fig. 13(b) shows a three-dimensional view of the LSB. The separation bubble is located between $X / c=0.4$ and $X / c=0.8$. The core of the vortex is located around $X / c \simeq 0.7$, while the reattachment point is hard to locate due to the time-averaging of the vortex shedding downstream of the LSB. The three-dimensional view moreover highlights that the LSB contour is not closed between the quasistable region and the unsteady one. Separation between these two regions still is however visible and located around $X / c \simeq 0.8$. This is confirmed by the skin friction coefficient along the chord at $Z / c=0.1$ shown in Fig. 14 (a) that reaches almost 0 at $X / c=0.75$ and then decreases rapidly in the unsteady region. The spanwise evolution of the skin friction coefficient shows however that only partial reattachment occurs around at $X / c=0.75$, whereas it fully reattaches at the trailing edge. Spatial fluctuations of the pressure coefficient are also observed in this region, as depicted in Fig. 14(b).

Table 2 provides the characteristics of the LSB along with comparisons against two sets of measurements from Burgmann et al. [18] and Burgmann and Schröder [35]. Separation $\left(x_{s}\right)$ and reattachment $\left(x_{r}\right)$ points are determined with the null skin friction coefficient, while the length of the $\operatorname{LSB}\left(l_{s}\right)$ is defined as the distance between those two points and its height $\left(h_{s}\right)$ as the maximum height obtained by the $\delta_{99}$ criterion. The present computation agrees well with experiments of Burgmann and Schröder [35]. Though the separation point is slightly advanced, the LSB length and height are within the range of the experimental values. As long as the reattachment point is concerned, full reattachment is observed at the trailing edge $(x / c=1)$, which was also found by 

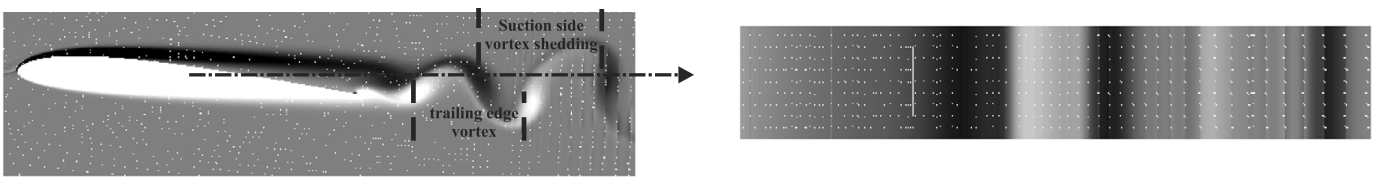

(a) $\operatorname{Re}=5000$

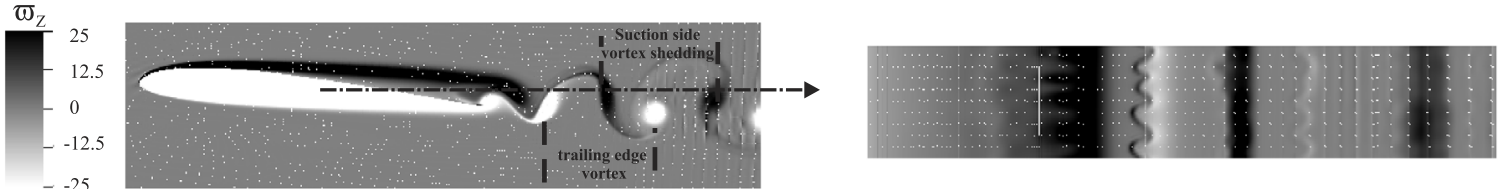

(b) $\operatorname{Re}=10000$

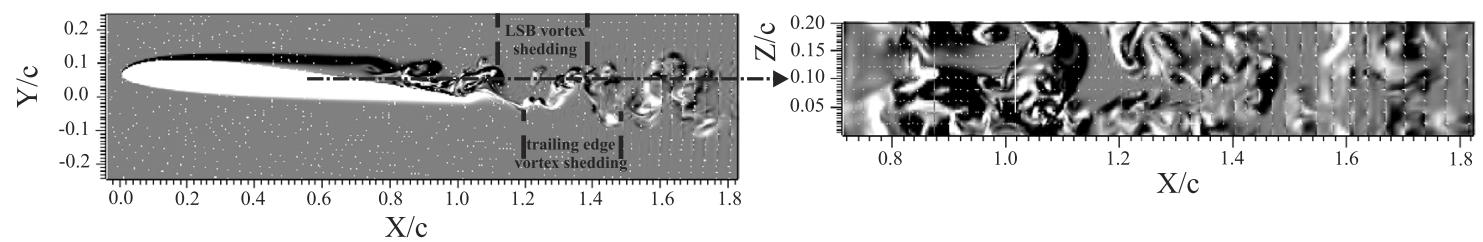

(c) $\operatorname{Re}=20000$

Fig. 12. Effect of Reynolds number on vorticity field, 3D computation, confined domain.

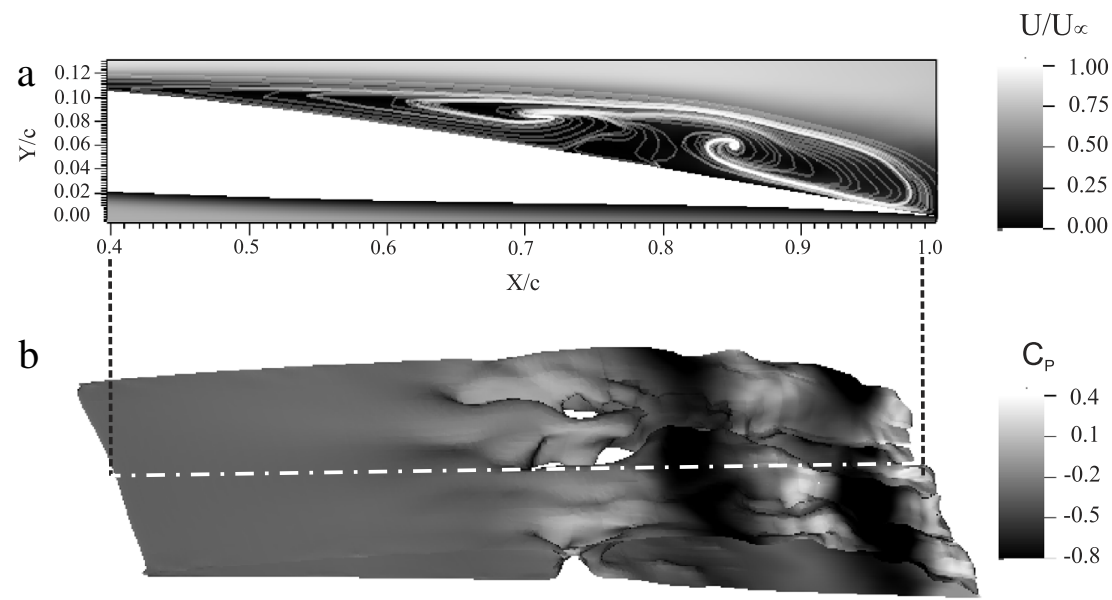

Fig. 13. (a) Time averaged velocity field and velocity streamlines near the foil trailing edge; (b) Time averaged iso surface of $U_{y} / U_{\infty}=0.05$ colored with the pressure coefficient, $R e=20000$.

a

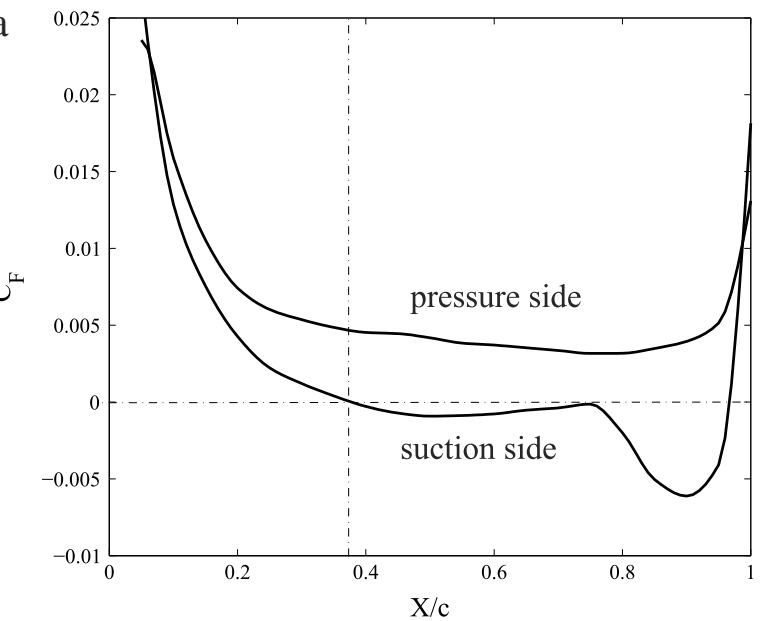

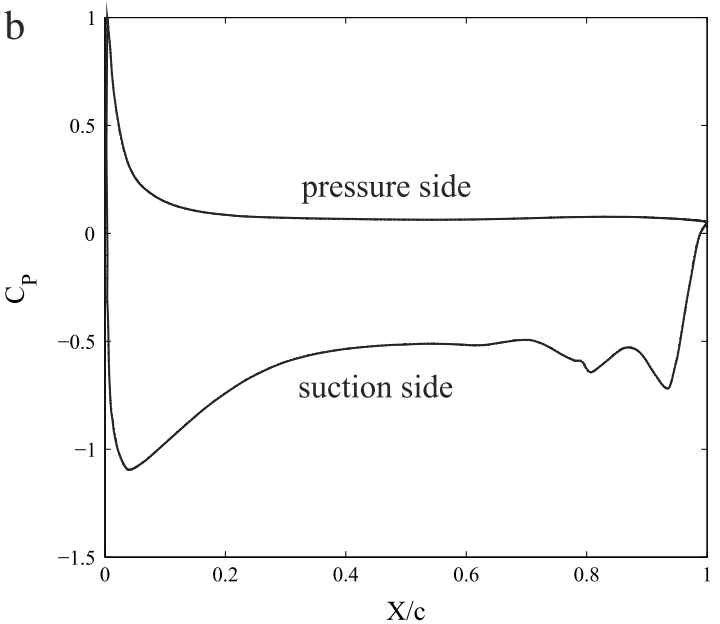

Fig. 14. (a) Friction coefficient, (b) Pressure coefficient, $R e=20000$. 


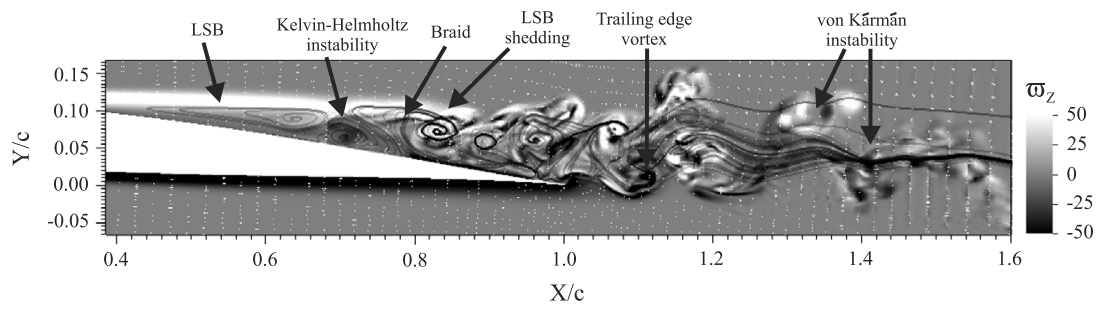

Fig. 15. Instantaneous vorticity field and velocity streamlines along the laminar to turbulent transition region, $R e=20000$.
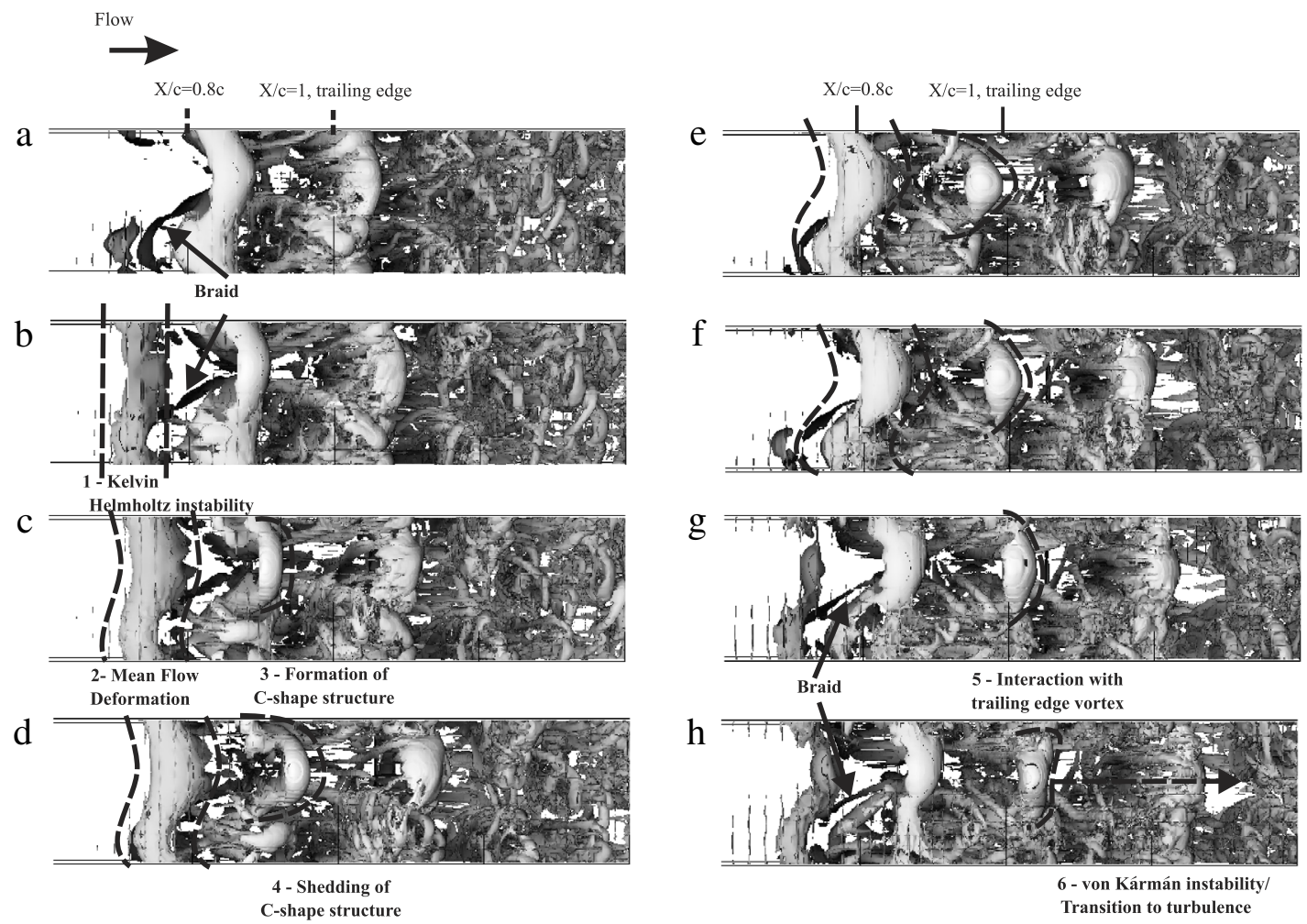

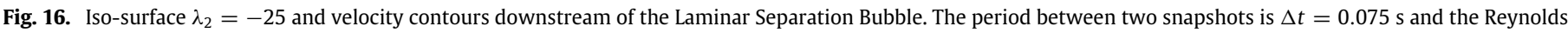
number is set to $R e=20000$.

the experiments of Olson et al. [20] and recently by Nati et al. [21] for a higher Reynolds number $(\operatorname{Re}=30000)$. Olson et al. [20] have also compared their results with Burgmann and Schröder [35] and Burgmann et al. [18]. They explain the discrepancies in the LSB characterization by uncertainties due to determination of the angle of attack, to uncertainties due to near-wall measurement resolution, as well as the sensitivity to the Reynolds number and the effects of free-stream turbulence. This last parameter has been investigated and it has been shown that increasing free-stream turbulence tends to slightly move upstream the reattachment point, consistent with the present calculation that does not have free-stream turbulence. Finally, the Strouhal number $S t_{\theta}$ used to characterize the LSB vortex shedding is close to the value $S_{t \theta}=$ 0.068 of Pauley et al. [9]. It has moreover been shown that this value has to be within the range 0.005-0.011.

\subsection{Overview of the laminar-turbulent transition at $R e=20000$}

As a first step toward our understanding of the laminarturbulent transition taking place at $R e=20000$, various structures and related phenomenon are identified from instantaneous flow visualizations. Fig. 15 depicts the instantaneous spanwise nondimensional vorticity field $\varpi_{Z}$ together with velocity streamlines from $X / c=0.4$ to $X / c=1.6$. Four events can be observed:
Table 2

LSB characteristics, comparison with PIV measurements. $x_{s}$ separation point, $x_{r}$ reattachment point, $l_{s}$ LSB length, $h_{s}$ LSB height, $S_{t \theta}=f \theta_{\text {sep }} /\left(u_{\text {sep }}\right)$ Strouhal number at separation.

\begin{tabular}{lllll}
\hline & DNS & $\begin{array}{l}\text { Burgmann and } \\
\text { Schröder [35] }\end{array}$ & $\begin{array}{l}\text { Burgmann } \\
\text { et al. [18] }\end{array}$ & $\begin{array}{l}\text { Olson } \\
\text { et al. [20] }\end{array}$ \\
\hline$\left(x_{s}\right)$ & $0.39 c$ & $0.4 c$ & $0.51 c$ & $0.4 c$ \\
$\left(x_{r}\right)$ & $1 c$ & $0.775 c$ & $0.83 c$ & $1 c$ \\
$\left(l_{s}\right)$ & $0.36 c$ & $0.375 c$ & $0.32 c$ & - \\
$\left(h_{s}\right)$ & $0.062 c$ & $0.0735 c$ & $0.053 c$ & - \\
$S_{t \theta}$ & 0.0073 & 0.0095 & 0.011 & - \\
\hline
\end{tabular}

- A classical laminar separation bubble lies between $X / c=0.4$ and $X / c=0.7$. It is made of a dead region that is stationary up to the vortex core, and a region of reattachment exhibiting low frequency fluctuations in-between $X / c=0.65$ and $X / c=0.8$.

- An almost two-dimensional vortex is shed from the LSB and is convected downstream.

- Due to the curvature of the wing and its angle of attack, the vortex extends in the wall-normal direction. Small scales can be observed, and the flow starts to transition.

- When reaching the trailing edge, the vortex seems to interact with a trailing edge vortex to form von Kármán vortices.

It appears from this visualization that an interaction might exist between two primary instabilities-e.g. the Kelvin-Helmholtz 

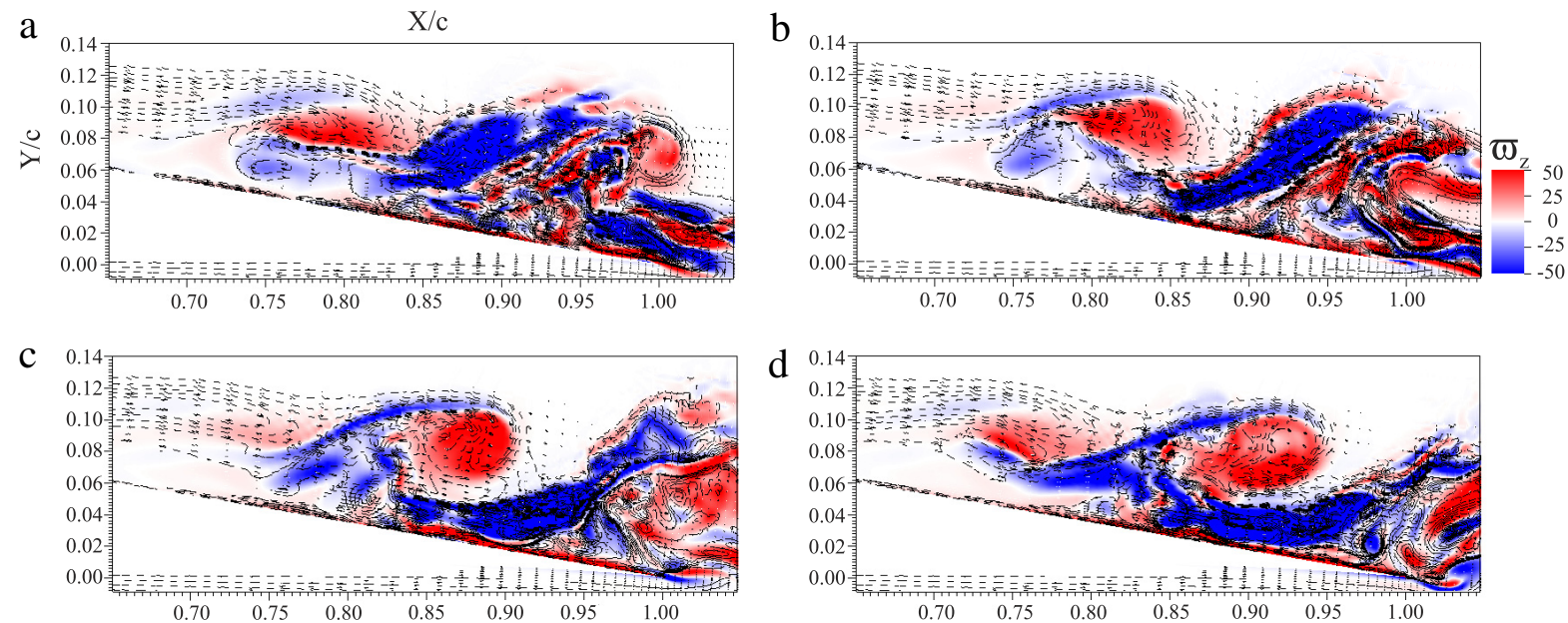

Fig. 17. Contours of $\varpi_{X}$ near the trailing edge, dotted lines (-- -) are iso- $\varpi_{Z},(X, Y)$ plan, $Z / c=0.15, R e=20000$.

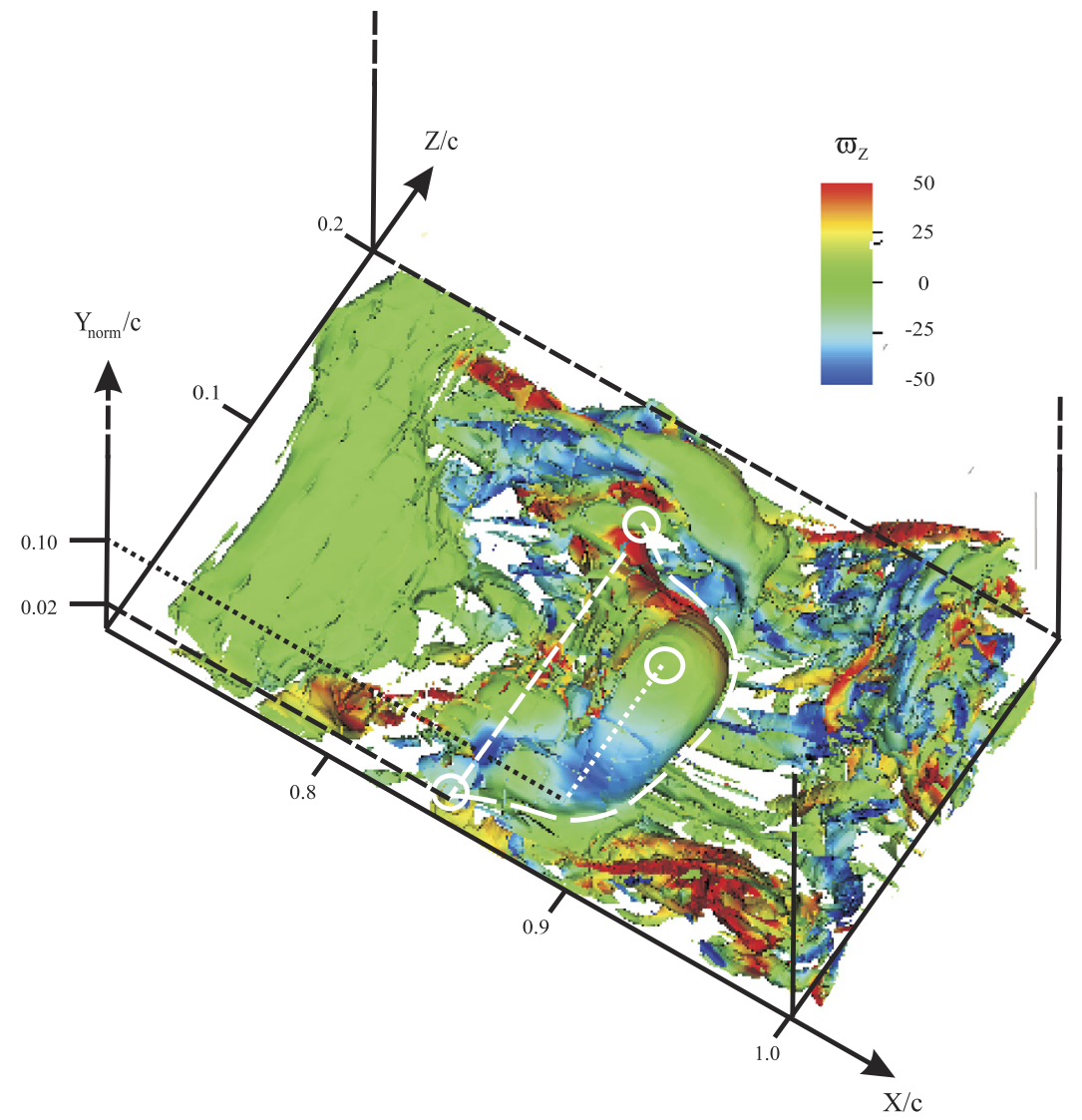

Fig. 18. Hairpin structure, surface of iso- $\lambda_{2}$ and contours of $\varpi_{Z}$ downstream of the Laminar Separation Bubble. $\lambda_{2}=-25, R e=20000$.

instability associated to the shedding of the LSB which occurs upstream of the trailing edge, and a von Kármán instability which takes place at the wing's trailing edge. Because these two instabilities generate very comparable vortical scales, they have the potential to interact with each other, hence possibly altering the physics of the LSB vortex shedding and the associated shedding frequency. This interaction will be further highlighted in Sections 4.4.1 and 4.4.2.

In order to get a better understanding of the development of the three-dimensional flow downstream of the LSB, $\lambda_{2}$ visualizations of the vortical structures within the flow at various instants of time are depicted in Fig. 16. These figures highlight how a two-dimensional vortex detaches from the LSB before being convected toward the trailing edge of the wing. During this convection, it moreover experiences a spanwise distortion eventually transforming the initial two-dimensional vortex into a $\mathrm{C}$-shaped one. The typical wavelength of this spanwise modulation is $\lambda / D=3$ (with $D$ being the diameter of the initial twodimensional vortex), in agreement with what has been found in [24]. Finally, as shown in Fig. 16(d)-(g), this C-shaped vortex gets more and more deformed as it approaches the trailing edge. Passed the trailing edge, it is not coherent enough anymore and eventually breaks down into small-scale structures in the wake.

Fig. 17 depicts the non dimensional vorticity $\varpi_{X}=\varpi_{X}^{\star} c / U_{\infty}$ from the LSB to the vicinity of the trailing edge. These figures 

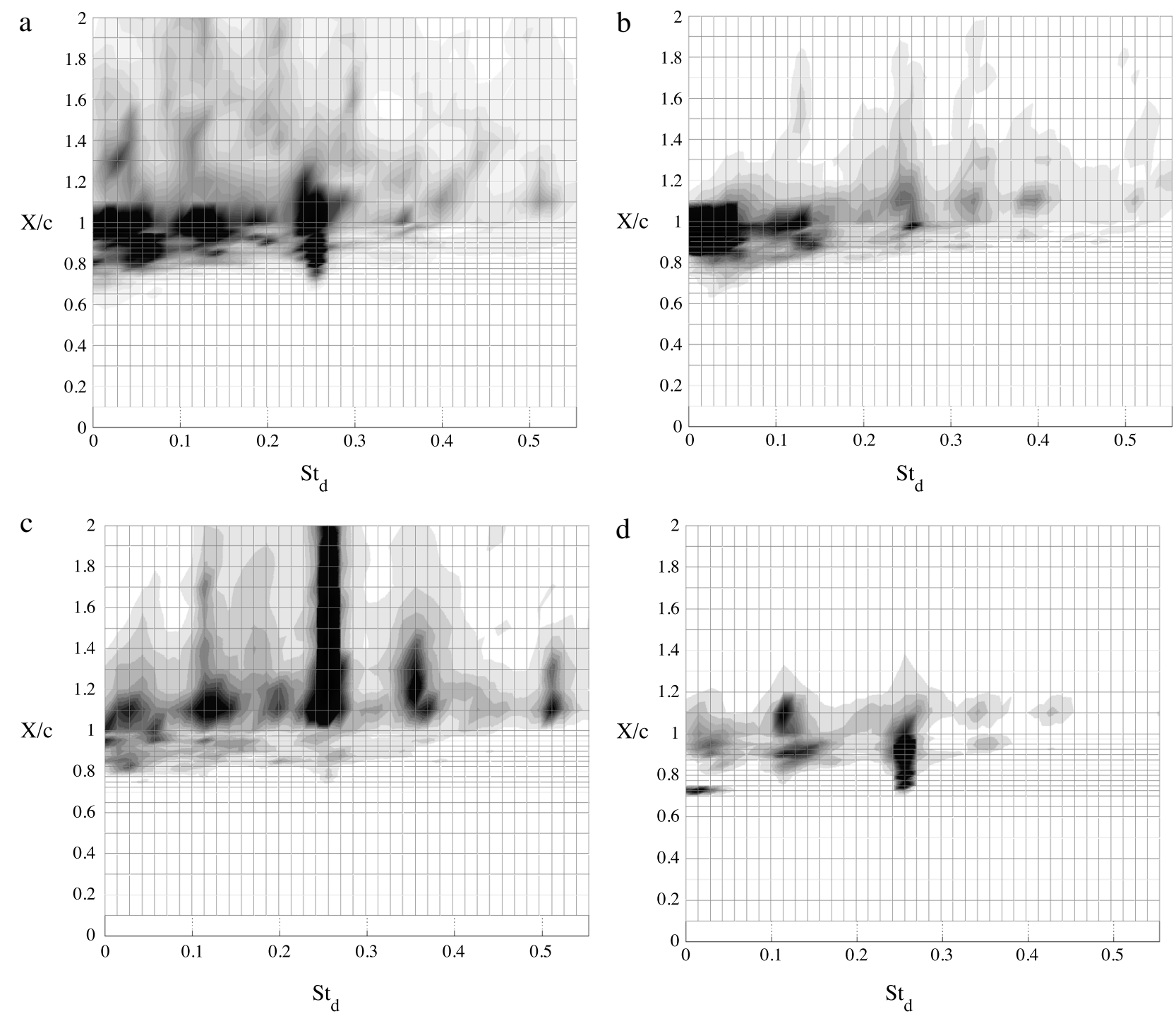

Fig. 19. Spectral analysis along the chord (a) $X$-velocity, (b) $Y$-velocity (c) $Z$-velocity (d) pressure, $R e=20000$.

clearly show that the initial two-dimensional vortex shedding from the LSB is related to the well-known Kelvin-Helmholtz instability. Indeed, classical shapes of Kelvin-Helmholtz waves are observed wherein positive regions of $\varpi_{X}$ develop in the core of the vortex and are convected in the streamwise direction, while negative regions of $\varpi_{X}$ develop between the Kelvin-Helmholtz vortex and the LSB, before rolling-up and causing the shedding of the vortical structure. As observed by Jones et al. [24], the development of the vorticity downstream of the laminar separation bubble directly depends on the destabilization of the vortical structure. They have observed a high level of streamwise vorticity $\varpi_{X}$ in the core of the vortex (due to elliptic instability) and in the braid region (due to hyperbolic instability). Finally, Fig. 18 is a close-up of Fig. 16(d) around the C-shaped vortex that results from the spanwise distortion of these Kelvin-Helmholtz waves. The same value of $\lambda_{2}$ is taken, colored with the spanwise vorticity $\varpi_{Z}$. Two vortex filaments, one on each side of the vortex, having opposite vorticity values $\varpi_{Z}$ are observed. These filaments are moreover located in the vicinity of the wall, around $Y / c=$ 0.02 , whereas the center of the vortex is significantly higher, around $Y / c=0.1$. Based on this description, this C-shaped structure appears quite similar to a hairpin vortex as defined by Adrian [37].

\subsection{Physical analysis}

\subsubsection{Spectral analysis}

Fig. 19 depicts the spectral density plots along the chord and in the wake for the three velocity components (see Fig. 19(a)-(c)) as well as for the pressure (see Fig. 19(d)). Each of these spectra highlights a different aspect of the dynamics of this transitional flow:

- The spectrum of velocity $U_{X}$ in the chordwise direction (Fig. 19(a)) exhibits the three components associated with flapping, shedding of LSB and von Kármán, where the largest amplitudes are located inside the transition region. The flapping and shedding frequencies disappear past the trailing edge, whereas the von Kármán frequency disappears around $X / c=$ 1.2 .

- The spectrum of $U_{Y}$ in Fig. 19(b) has a dominant component associated with the von Kármán instability at a Strouhal $S t_{d}=$ 0.25 . A lower amplitude component is also observed at $S t_{d}=$ 0.12 and corresponds to the LSB shedding. Harmonics of these two frequencies are also observed. It has to be noted that all the frequencies are located in the wake, and the spectra are mostly flat in the transition region. This is because of the influence of the wall that blocks the $U_{Y}$ fluctuations up to the wing surface, 


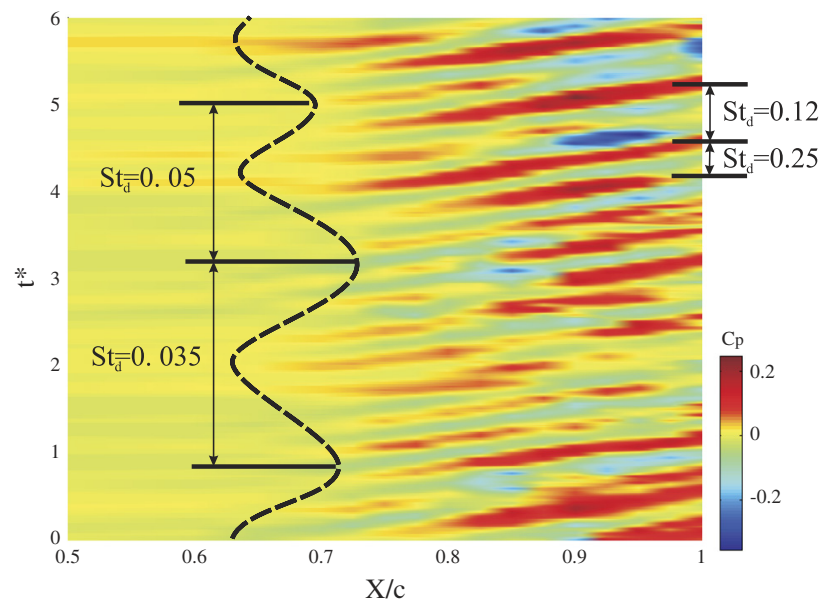

Fig. 20. Pressure coefficient along the chord as function of time, $R e=20000$.

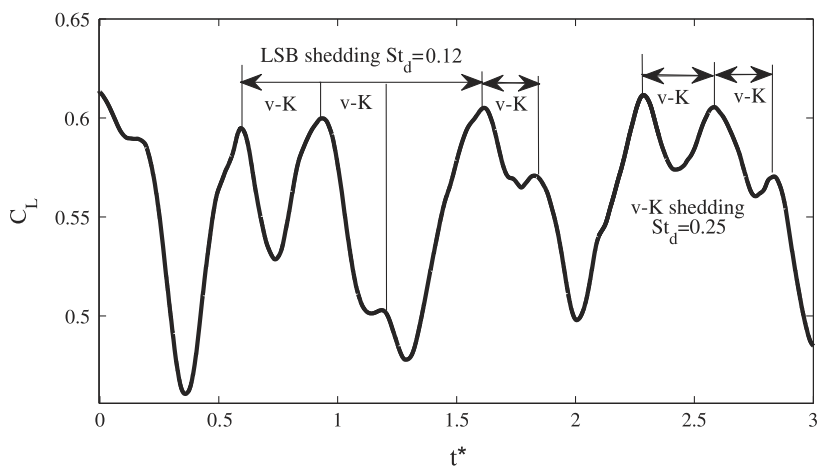

Fig. 21. Lift coefficient as function of time, $R e=20000$.

whereas it is the main direction of the fluctuation of the von Kármán vortices once it convects in the wake.

- The $U_{Z}$ spectrum in Fig. 19(c) displays large amplitudes only for the lower frequency associated to flapping. This observation suggests that the LSB reattachment may have a three-dimensional behavior, and that three-dimensional effects appear directly in the unsteady region of the LSB, which is confirmed by the observations in Figs. 12 and 16.

- The spectrum of pressure in Fig. 19(d) shows that the frequency associated to the von Kármán instability has a large amplitude in the transition region between $X / c=0.75$ and $X / c=1$.
A peak with low frequency is also observed at $X / c=0.725$, associated with the flapping of the LSB.

As shown in Fig. 20, pressure waves propagate in the flow direction, starting from approximately $X / c=0.7$ down to the trailing edge. Fig. 19(d) highlights that the dominant Strouhal number is associated with the von Kármán frequency $\left(S t_{d} \approx\right.$ 0.25 ), and the Strouhal associated to LSB vortex shedding is mainly observed near the trailing edge $\left(S t_{d} \approx 0.12\right)$. These two frequencies seem physically independent, while the subharmonic behavior suggests that the LSB vortex shedding is locked-in the von Kármán instability. Fig. 20 also shows a very low Strouhal number around the reattachment region which could be associated with the flapping of LSB.

The time-evolution of the lift coefficient shown in Fig. 21 clearly highlights the coupling between the two primary instabilities. The main LSB Strouhal shedding frequency is observed around $S t_{d} \approx 0.12$ with relatively large amplitude, while the von Kármán appears inside the LSB shedding period with lower amplitude.

\subsubsection{Dynamic Mode Decomposition}

Dynamic Mode Decomposition is used as a tool to provide a space-time physical analysis of the laminar-turbulent transition occurring along the wing at $R e=20000$. It enables us to link a spatial structure (a Dynamic mode, also called Koopman mode) to a given frequency and to compare with the spectral analysis performed in Section 4.4.1. Present DMD results are based on a sequence of 300 velocity field snapshots sampled at a constant sampling period $\Delta t=0.02$. This sampling period discretizes each period of the von Kármán vortex shedding (i.e. $S t_{d}=0.25$ ) with 17 snapshots. The eigenspectrum of the resulting Koopman operator is shown in Fig. 22(a). Fig. 22(b) provides the amplitude of each Koopman mode with respect to the corresponding frequency. Whereas only one frequency dominates the dynamics at $R e=$ 10000 (see Fig. 10), two frequencies are clearly dominating the dynamics at $R e=20000: S t_{d} \simeq 0.12$, associated to transition, and $S t_{d} \simeq 0.24$, which is characteristic of the von Kármán vortex shedding, already observed at lower Reynolds numbers. It has to be noted furthermore that the low-frequencies also provide a nonnegligible contribution to these dynamics, though they are subdominant. Fig. 23 depicts the spatial distributions of three modes of interest, identified from Fig. 22(b), in given $(x, y)$ and $(x, z)$ planes. It is clear that all three modes have large amplitudes in the LSB region (i.e. $0.8 \leq X / c \leq 1$ ), further highlighting the crucial importance of the transition process in the flow dynamics.
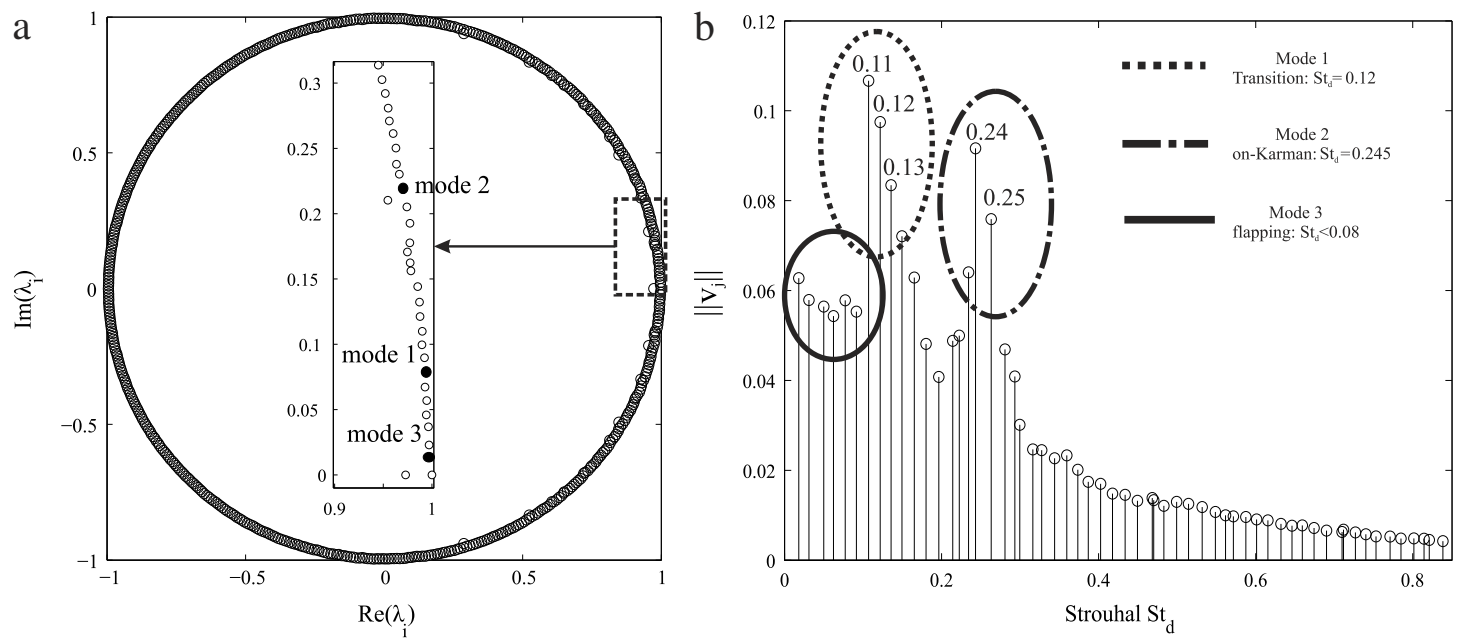

Fig. 22. Dynamic Mode Decomposition, (a) eigenvalues along the unit circle, (b) Koopman spectrum, $R e=20000$. 

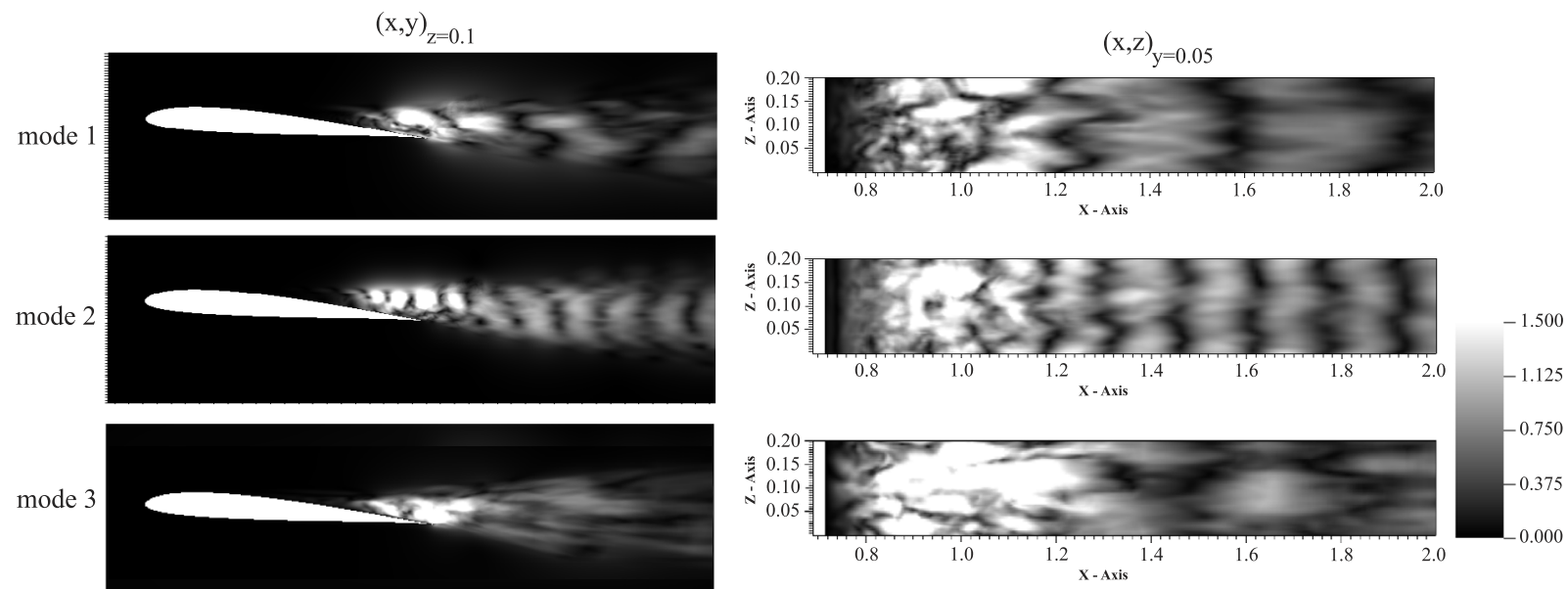

Fig. 23. Velocity field $\|U\|$ for the 3 first modes. $(X, Y)$ planes are taken at mid $\operatorname{span}(Z=0.1) ;(X, Z)$ planes are taken at the LSB level $(Y=0.05)$. Re $=20000$.

The spatial distribution of the leading Koopman mode associated to the LSB vortex shedding is depicted in Fig. 23 (see mode 1), along with that of the Koopman mode associated to the von Kármán vortex shedding (see mode 2 ). As illustrated by these two figures, it is clear that the mode associated to LSB vortex shedding has a dominant streamwise wavelength twice as large as that of the von Kármán vortex shedding. This factor 2 in the dominant wavelength as well as in the associated Strouhal numbers suggests that the LSB vortex shedding interacts with the von Kármán instability and is locked-in as one of its subharmonics. Moreover, comparison of the von Kármán mode at $R e=20000$ with the one obtained at $R e=10000$ (see Fig. 10) further highlights this intricate interaction between the two types of vortex sheddings. Indeed, in the laminar case ( $R e=10000$ ), the von Kármán instability is visible only past the trailing edge of the foil $(X / c=1)$, while in the transitional case $(R e=20000)$, it clearly has a non-zero spatial distribution along the wing, up to $X / c \simeq 0.75$. As shown in Fig. 16 , the region $0.75<X / c<1$ is the region where the boundary-layer flow along the foil starts to become unsteady and where coherent transitional structures begin to appear, i.e. where the LSB vortex shedding takes place.

It is then useful to observe the modes patterns in the three directions of space, and to spatially correlate them with the spectral analysis in Fig. 19. Figs. 24-26 show the $U_{X}, U_{Y}$ and $U_{Z}$ velocity components for the modes 1,2 and 3 , respectively. Three views are shown for each component: a three-dimensional view with isosurface of velocity normalized between -1 and +1 and the corresponding top view in the spanwise direction in order to show the shape of each mode. A colorplot of the mode's velocity in the $Y / c=0.05$ plane is also shown in order to observe the amplitudes. Similarities between mode 1 and 2 are evident, confirming that the mode 1 of larger amplitude is associated with an harmonic of the von Kármán mode. As a consequence, a classic, 2D shape of the von Kármán mode is observed for $V_{X}$ and $V_{Y}$ in Figs. 24 and 25 with wavelength twice larger for mode 1 than for mode 2. This wavelength is smaller on the foil surface due to higher velocity convection. The $U_{Z}$ velocity shows that the patterns get globally three-dimensional right after the reattachment point at $X / c=0.8$. Whereas the von Kármán mode in Fig. 25 is almost twodimensional, it appears that the mode 1 has three-dimensional multi-scaled patterns that start from the reattachment point of the LSB and may correspond to the formation of vortex filaments around $X / c=0.7$ in the braid region. The velocity $U_{Y}$ in Fig. 24(b) exhibits similar patterns. This analysis confirms that (i) mode 1 is associated with the LSB vortex shedding and (ii) this shedding process is locked-in with the von Kármán mode. a
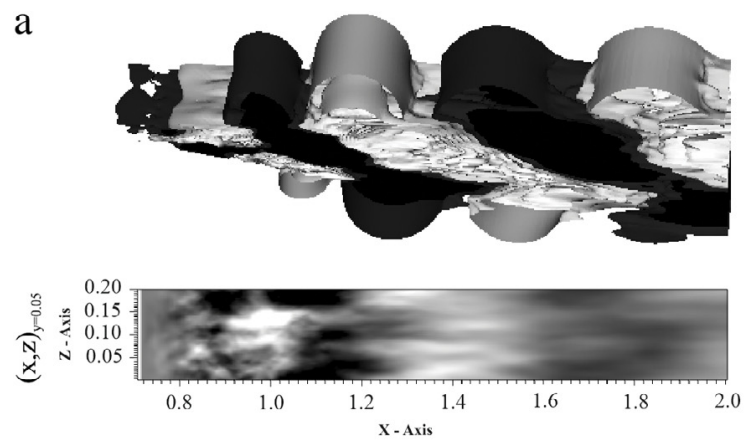

b
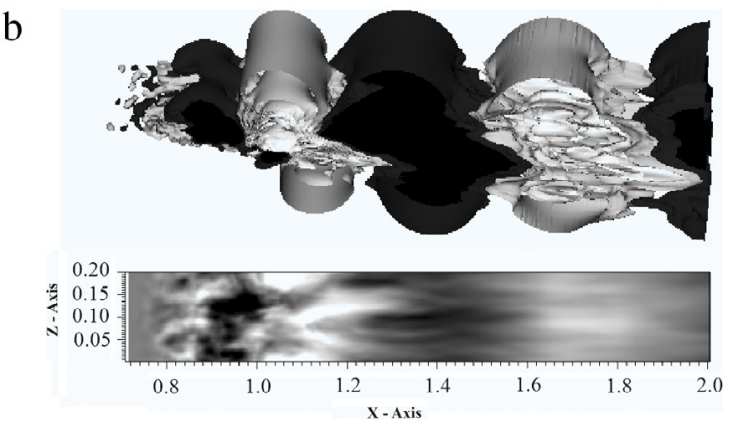

C

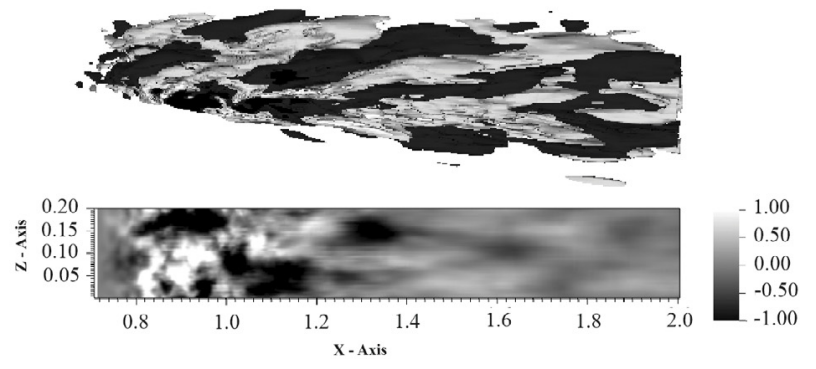

Fig. 24. Iso contours of velocity and velocity field in $(x, z)$ plan, (a) $V_{X}$, (b) $V_{Y}$, (c) $V_{Z}$ for the mode $1 . R e=20,000$.

The lower frequency mode 3 shows early amplitudes between $0.6 \leq X / c \leq 0.8$ that could correspond to the LSB flapping. The $(x, z)$ plans of $U_{X}$ and $U_{Z}$ show that the modulation is mostly in the spanwise direction, confirming that the reattachment and the flapping motion are globally three-dimensional. Despite its amplitude being maximum within the transitional region, it is clear from its spatial distribution in the wake that this mode does not correspond to some sort of vortex shedding. Hence, in order to identify the type of structures associated to this mode, the motion 
a
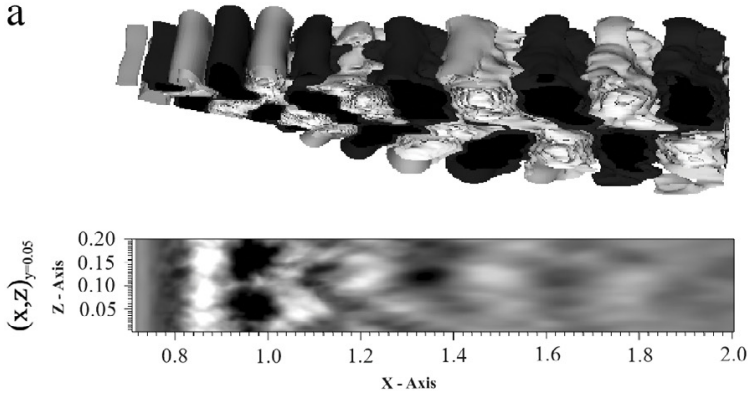

b
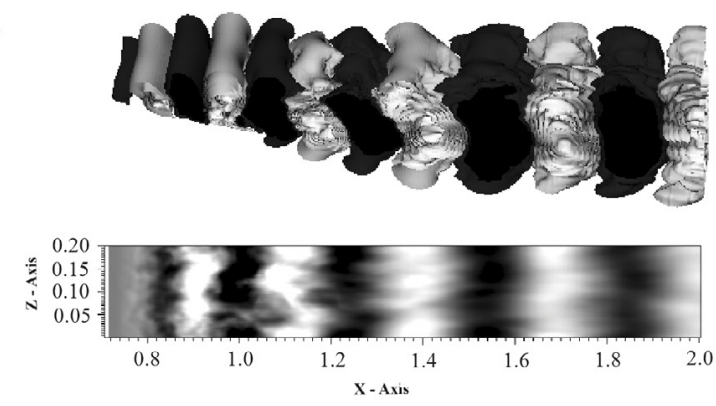

c
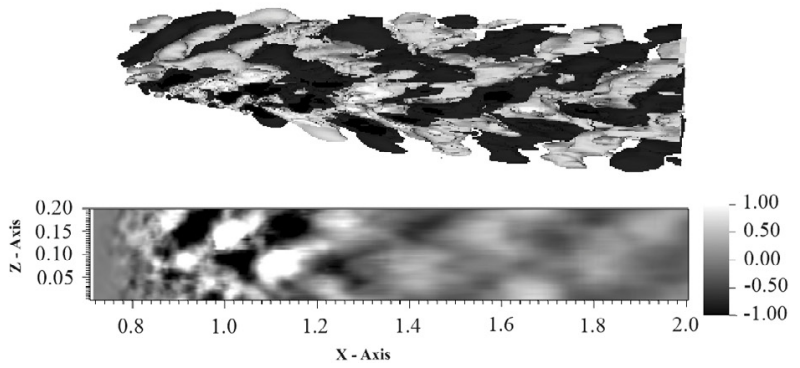

Fig. 25. Iso contours of velocity and velocity field in $(x, z)$ plan, (a) $V_{X}$, (b) $V_{Y}$, (c) $V_{Z}$ for the mode $2 . R e=20,000$.

it induces in various $(y, z)$ planes along the chord is depicted in Fig. 27. It is clear from this figure that the associated structures are pairs of chordwise counter-rotating vortices that, due to the lift-up effect [38], promote chordwise low- and high-speed streaks. These streaks eventually induce a spanwise modulation of the reversed flow region which might explain the low-frequency oscillation of the pressure coefficient depicted in Fig. 20. A possible explanation of the physical origin of these streaks can be found in the work of Rodriguez and Theofilis [39]. By means of linear stability analyses, the former authors have shown that, beyond a given Reynolds number, LSB can experience a centrifugal global instability yielding to its spanwise modulation. Moreover, the associated global mode typically consists in pairs of streamwise-oriented counter-rotating vortices inducing streamwise velocity modulations through the lift-up effect.

\section{Discussion}

The results presented in this paper lead to the following discussion on the transition mechanisms at $R e=20000$. Higher Reynolds number cases have been investigated in the literature, particularly the case $R e=60000$ shown in Fig. 1(d). For this Reynolds number, DNS calculations and measurements have been performed for the SD7003 section in [22,17] and similar DNS calculation is presented in [24] for the NACA0012. In all cases, flapping of the LSB is associated to a low frequency modulation of the most amplified disturbance, and Kelvin-Helmholtz instabilities are observed and lead to the formation of vortices in the shear layer. The development of turbulence downstream the bubble is however still the subject of many discussions, and depends on a
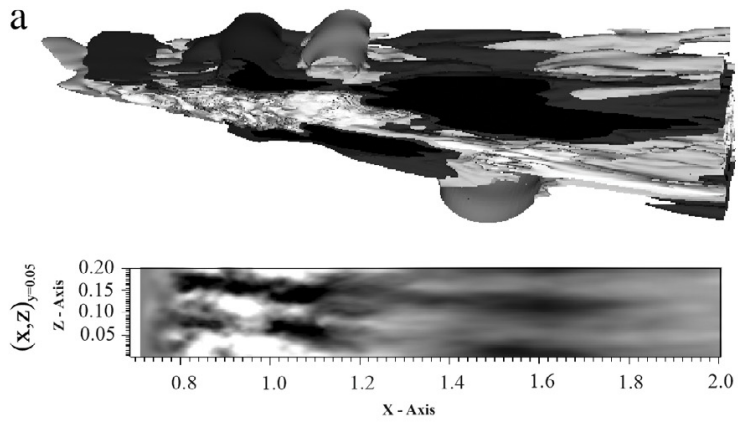

b
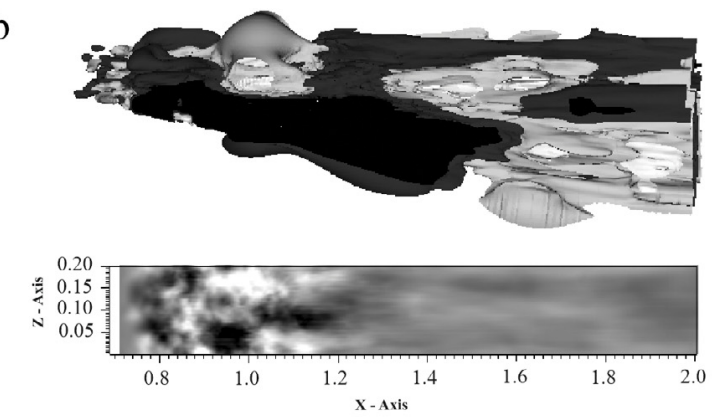

$\mathrm{C}$
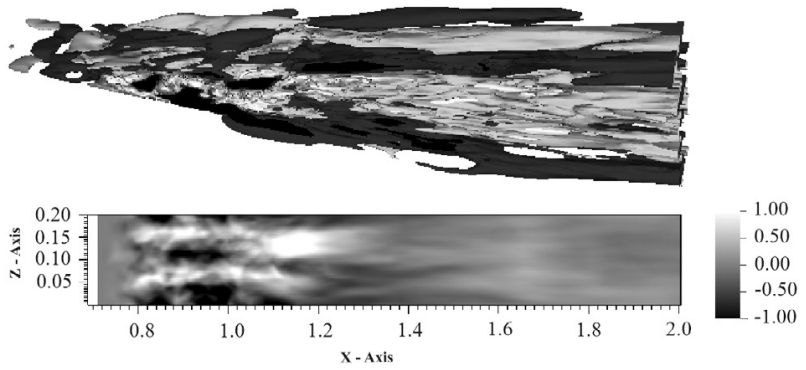

Fig. 26. Iso contours of velocity and velocity field in $(x, z)$ plan, (a) $V_{X}$, (b) $V_{Y}$, (c) $V_{Z}$ for the mode $3 . R e=20,000$.

the type of analyses performed. The numerical works of Jones et al. [24] find that the flow is not fully attached nor fully separated downstream of the LSB because of the presence of coherent structures all along the chord downstream the transition that induces large fluctuations of the skin friction coefficient. In the mean time, they observed spanwise coherence of the vortical structures. Hain et al. [17] state that full turbulent reattachment occurs around the mid chord, whereas a weak coherence is observed in the spanwise direction. It seems obvious that this difference in the physics mainly depends on the Reynolds number and on the geometry of the wing, where the leading edge geometry, the camber and the angle of attack can change the critical Reynolds number that can modify the location and the behavior of transition along the wing. The Reynolds number investigated in this paper is significantly lower ( $R e=20000)$, but the transition process seems to be closer to the DNS of Jones et al. [24] where the foil section has no camber and transition is triggered using volume forcing. It is clear that the LSB obtained corresponds to a long bubble with no full reattachment of the LSB. The main difference is the location of transition that is closer from the trailing edge where the Kelvin-Helmholtz vortices interact with the wake flow. This interaction has not been investigated previously (see the work of Burgmann and Schröder [35] at a similar Reynolds number that only consider the foil in their measurements).

The transition mechanisms can be described as follows. The transition is clearly dominated by Kelvin-Helmholtz instabilities and the growth of two-dimensional vortices in the shear layer. These vortices then deform upstream and are shed downstream of the LSB, which has been observed in most of the studies related 


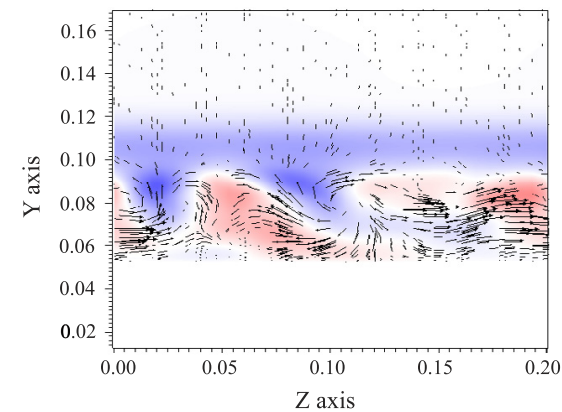

(a) $\mathrm{X} / \mathrm{c}=0.70$

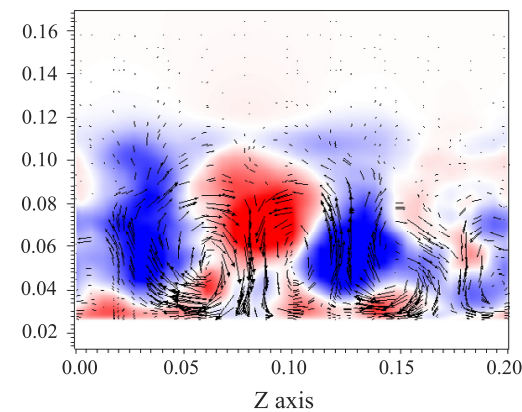

(b) $\mathrm{X} / \mathrm{c}=0.85$

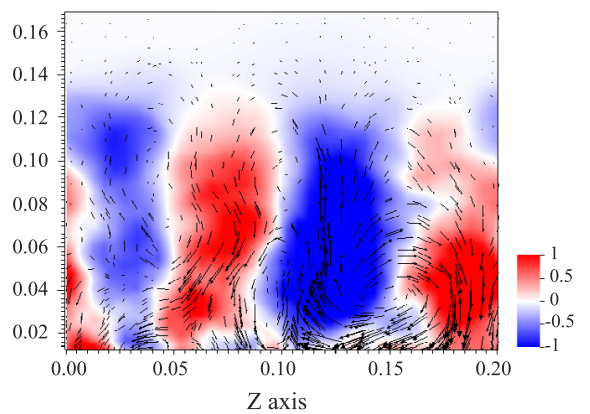

(c) $\mathrm{X} / \mathrm{c}=1.00$

Fig. 27. Velocity field $U_{X}$, and vector plots $\left(U_{Y}, U_{Z}\right)$ for the mode 3 at different chordwise locations. $R e=20000$.

to laminar to turbulent transition on wing sections. Whereas the DMD mode associated to these Kelvin-Helmholtz instabilities has the largest amplitude compared to the other modes, the frequency associated with this mode is driven by the von Kármán mode (mode 2). On the one hand, the von Kármán Strouhal number of vortex shedding stays constant between the fully laminar $(R e=5000$ and $R e=10000)$ and the weakly transitional case $R e=20000$ meaning that this instability develops almost independently from other physical mechanisms. On the other hand, the Strouhal number of LSB shedding behaves almost as a subharmonic of the von Kármán mode. It is suggested in this paper that the natural LSB shedding frequency is near the subharmonic of the von Kármán shedding frequency and partial lock-in phenomenon is occurring. The main conclusion is that the first stage of the transition process seems to be physically independent from the wake flow (i.e. generation of Kelvin-Helmholtz instability and the development of the three-dimensional flow), whereas the LSB shedding process seems highly dependent on the von Kármán instability, with the highest von Kármán modal amplitudes found in the transition region between $0.8 \leq X / c \leq 1$. The von Kármán instability can stabilize the transition to turbulence process and maintain the coherence of the flow from the unsteady region down to the trailing edge, and transition to turbulence mainly occurs in the wake of the wing due to the instability of the wake.

\section{Conclusion}

In this study, the transition to turbulence over a wing section is investigated numerically using direct numerical simulations performed with the code NEK5000. The wing has a SD7003 section and the transitional Reynolds number $R e=20000$ with an angle of attack $\alpha=4^{\circ}$ has been chosen. The mechanisms yielding transition to turbulence have been studied by the joint use of space-time analysis of the flow physics and Dynamic Mode Decomposition.

Low Reynolds number simulations were first performed $(R e=$ 5000 and $R e=10000$ ), where two-dimensional von Kármán vortices are observed in the wake with a characteristic Strouhal number based on the wing thickness of $S t_{d}=0.238$. The case at $R e=20000$, also shows von Kármán like vortices together with complex transition mechanism. The first stage is highlighted by Kelvin-Helmholtz instability that occurs in the unstable region of the LSB. The vortex is first two-dimensional, then deforms to a C-shape structure and sheds when vortex filaments in the braid region break, which is associated with LSB vortex shedding. Because the structure is still coherent when it reaches the trailing edge, it interacts with a trailing edge vortex and is locked-in the wake instability with a frequency close to a subharmonic of the von Kármán frequency (i.e. $S t_{d}=0.12$ ).
A space time frequency analysis is then performed. Three frequencies with distinct physical mechanisms are observed. First, the Strouhal number of the von Kármán vortex shedding $S t_{d}=$ 0.248 appears to be very similar to the lower Reynolds number case $\left(R e=10000, S t_{d}=0.238\right)$. It is however observed that this frequency appears upstream of the trailing edge compared to the $R e=10000$ case, i.e. in the transition region, confirming the interaction with the LSB shedding frequency. The LSB vortex shedding Strouhal frequency $\left(S t_{d}<0.12\right)$ appears a little earlier along the chord and vanishes almost completely past the trailing edge. It is also shown that, though these frequencies are lockedin, they are spatially independent. The spectrum also shows a third lower frequency $\left(S t_{d}<0.08\right)$ associated with the flapping of the LSB, and occurring upstream of the two other frequencies, where a low frequency pressure fluctuation is observed around the reattachment point.

Dynamic Mode Decomposition is then used to identify spatial structures to the different Strouhal frequencies. The three characteristics frequencies are observed, where the maximum amplitude is found for the LSB vortex shedding. A classical von Kármán mode is found at $S t_{d}<0.248$, and is fully two-dimensional along the chord, and tends to get three-dimensional in the wake. The lower Strouhal $S t_{d}<0.12$ confirms that it can be associated to a subharmonic of the von Kármán mode. The later seems to have a destabilizing effect on the transition process. Finally, the mode of LSB flapping (mode 3 ) results from the development of streaks that could be responsible for the first destabilization of the flow.

It has to be noted that whereas the LSB characteristics and the transition process were already investigated experimentally and were used, when available for validation of the DNS calculations, the present study highlights some physical aspects that are still waiting experimental confirmation. In particular, the interaction and possible lock-in of the LSB shedding with the wake flow has not been investigated experimentally for this case because of control volumes used in the PIV and LDV measurements being usually limited to the airfoil. Experimental confirmation of this particular point could then enhance the understanding of transition mechanisms in the case where it is located near a periodic wake flow.

\section{Acknowledgments}

Most of the computations have been performed on the IBM x3750 (ADA) at GENCI/IDRIS, Orsay, France. Flow visualizations have been made using the open-source software VisIt [40].

\section{Appendix. The Dynamic Mode Decomposition method}

Transitioning and turbulent flows exhibit complex phenomena involving a substantially large range of both spatial and temporal scales. Because of this complexity, analyzing and inferring conclusions about the dynamics and underlying mechanisms directly 
from raw measurements is hardly feasible. As to unravel some order hidden behind this apparent complexity, a modal decomposition of the data is usually performed. Various decompositions can be employed: Proper orthogonal decomposition [41,42], global eigenmodes [43] and balanced modes [44] are the most commonly used. The aim of such decompositions is to identify coherent structures in order to reduce the large scale dynamics to a smaller number of degrees of freedom. Identifying these coherent structures also allows to investigate their spatio-temporal characteristics and have a better understanding of the patterns observed in the flow fields.

The decomposition employed in the present work has been introduced in 2008 by Schmid and Sesterhenn [45] as Dynamic Mode Decomposition (DMD) and Rowley et al. [46] as Koopman Mode Decomposition. Since then, other variations of this decomposition have been introduced. The algorithm presented here is the one originally introduced by Rowley et al. [46]. Assume one has a discrete set of flow observables $\mathbf{x}_{j}=\mathbf{x}(j \Delta t)$ at $k$ different time steps such that:

$\mathbf{K}=\left[\mathbf{x}_{0}, \mathbf{x}_{1}, \ldots, \mathbf{x}_{k-1}\right]$.

Koopman modes decomposition relies on the assumption that this sequence can be approximated by the following time-discrete linear dynamical system:

$\mathbf{x}_{i+1}=\mathbf{A} \mathbf{x}_{i}$.

As for global stability analysis of three-dimensional flows, the operator A might not be directly accessible or known (e.g. when the observables $\mathbf{x}_{i}$ are those of a non-linear flow). However, based on the assumption that it has been generated from the linear dynamical system (3), the sequence $\mathbf{K}$ can be seen as a Krylov sequence resulting from repeated application of the linear operator $\mathbf{A}$ onto the initial vector $\mathbf{x}_{0}$. Hence, given this Krylov sequence, one can construct a low-dimensional approximation of the operator $\mathbf{A}$ and compute reasonable approximations of its eigenvalues and eigenvectors. Difference with the global eigenmodes decomposition relies in the fact that, for Koopman modes decomposition, the matrix resulting from the low-dimensional approximation is not an upper Hessenberg matrix but a Companion one. Let us assume that the $k$ th vector of the Krylov sequence can be expressed as a linear combination of the previous iterates:

$\mathbf{x}_{k} \simeq c_{0} \mathbf{x}_{0}+c_{1} \mathbf{x}_{1}+\cdots+c_{k-1} \mathbf{x}_{k-1}$

$\mathbf{x}_{k} \simeq \mathbf{K c}$

where $\mathbf{c}$ is a column vector containing the $k$ coefficients $c_{i}$ needed for the linear combination. This linear problem can be recast into the following equivalent matrix form:

$\mathrm{AK} \simeq \mathrm{KC}$

where $\mathbf{C}$ is a $k \times k$ Companion matrix given by:

$\mathbf{C}=\left[\begin{array}{ccccc}0 & 0 & \cdots & 0 & c_{0} \\ 1 & 0 & & 0 & c_{1} \\ 0 & 1 & & 0 & c_{2} \\ \vdots & & \ddots & & \vdots \\ 0 & 0 & \cdots & 1 & c_{k-1}\end{array}\right]$.

The coefficients $c_{i}$ can be computed by solving a linear system or by a least-square procedure. It is clear from the structure of expression (5) that, apart from the specific structure of $\mathbf{C}$, a striking resemblance exists between the Koopman modes and the global mode decompositions. The Koopman modes decomposition can in fact be seen as a generalization of global stability analysis applied to a non-linear system. As a consequence, the eigenpairs $\left(\Sigma_{c}, \mathbf{X}\right)$ of the Companion matrix $\mathbf{C}$ are a reasonably good approximation to the eigenpairs $(\Sigma, \mathbf{U})$ of the operator $\mathbf{A}$ and one has:

$\left\{\begin{array}{l}\Sigma \simeq \Sigma_{c} \\ \mathbf{U} \simeq \mathbf{K X}\end{array}\right.$

The growth rate $\sigma$ and circular frequency $\omega$ of the Koopman modes can be recovered by:

$\sigma+i \omega=\frac{\log \left(\Sigma_{c}\right)}{\Delta t}$

$\Delta t$ being the sampling period between two snapshots of the observables. Because DMD is a data-driven decomposition, the sampling period $\Delta t$ of the snapshots sequence has to obey the Nyquist criterion, i.e. at least four snapshots per frequencies one wants to resolve. For further details on the algorithm, the reader is referred to [46] as well as [47]. It has to be noted finally that, while POD identifies spatial modes being orthonormal one to another, Koopman and DMD identify spatial modes which are each associated to a single frequency as underlined by its relationship to eigenvalue decomposition. Moreover, this decomposition is also close to discrete Fourier transform (DFT) and the two are strictly equivalent if the mean is subtracted from the initial data [48]. An important difference, however, is that DMD may potentially be genuinely non-normal. This non-normality may be crucial to capture dynamical effects such as transients or unstable phenomena. This enables DMD to identify and resolve instabilities and self-sustaining cycles with complex multiscale behavior.

\section{References}

[1] M. Genç, I. Karasu, H.H. Açikel, M.T. Akpolat, Low Reynolds number aerodynamics and transition, in: Tech, ISBN, 2012, pp. 978-953.

[2] M. Gaster, The structure and behaviour of laminar separation bubbles, AGARD CP 4 (1966) 813-854

[3] I. Tani, Low-speed flows involving bubble separations, Prog. Aerosp. Sci. 5 (1964) 70-103

[4] A. Dovgal, V. Kozlov, A. Michalke, Laminar boundary layer separation: instability and associated phenomena, Prog. Aerosp. Sci. 30 (1) (1994) 61-94.

[5] S.K. Roberts, M.I. Yaras, Large-eddy simulation of transition in a separation bubble, J. Fluids Eng. 128 (2) (2006) 232-238.

[6] O. Marxen, M. Lang, U. Rist, Vortex formation and vortex breakup in a laminar separation bubble, J. Fluid Mech. 728 (2013) 58-90.

[7] B. Armaly, F. Durst, J. Pereira, B. Schoenung, Experimental and theoretical investigation of backward-facing step flow, J. Fluid Mech. 127 (1983) 473-496.

[8] M. Alam, N.D. Sandham, Direct numerical simulation of short laminar separation bubbles with turbulent reattachment, J. Fluid Mech. 410 (2000) $1-28$.

[9] L.L. Pauley, P. Moin, W.C. Reynolds, The structure of two-dimensional separation, J. Fluid Mech. 220 (1990) 397-411.

[10] O. Marxen, D. Henningson, The effect of small-amplitude convective disturbances on the size and bursting of a laminar separation bubble, J. Fluid Mech. 671 (2011) 1-33.

[11] D.K. Walters, J.H. Leylek, Computational fluid dynamics study of wake-induced transition on a compressor-like flat plate, J. Turbomach. 127 (1) (2005) 52-63.

[12] B. Abu-Ghannam, R. Shaw, Natural transition of boundary layers-The effects of turbulence, pressure gradient, and flow history, J. Mech. Eng. Sci. 22 (5) (1980) 213-228.

[13] F.R. Menter, R. Langtry, S. Völker, Transition modelling for general purpose CFD codes, Flow Turbul. Combust. 77 (1) (2006) 277-303.

[14] M.S. Genç, İ Karasu, H.H. Açıkel, An experimental study on aerodynamics of naca2415 aerofoil at low re numbers, Exp. Therm Fluid Sci. 39 (2012) 252-264.

[15] I. Karasu, M. Genç, H. Açikel, Numerical study on low Reynolds number flows over an aerofoil, J. Appl. Mech. Eng. 2 (2013) 131.

[16] A. Ducoin, J.A. Astolfi, F. Deniset, J.F. Sigrist, Computational and experimental investigation of flow over a transient pitching hydrofoil, Eur. J. Mech. B Fluids 28 (2009) 728-743.

[17] R. Hain, C. Kähler, R. Radespiel, Dynamics of laminar separation bubbles at lowReynolds-number aerofoils, J. Fluid Mech. 630 (2009) 129-153.

[18] S. Burgmann, J. Dannemann, W. Schröder, Time-resolved and volumetric PIV measurements of a transitional separation bubble on an sd7003 airfoil, Exp. Fluids 44 (4) (2008) 609-622.

[19] W. Zhang, R. Hain, C.J. Kähler, Scanning PIV investigation of the laminar separation bubble on a sd7003 airfoil, Exp. Fluids 45 (4) (2008) 725-743.

[20] D.A. Olson, A.W. Katz, A.M. Naguib, M.M. Koochesfahani, D.P. Rizzetta, M.R. Visbal, On the challenges in experimental characterization of flow separation over airfoils at low Reynolds number, Exp. Fluids 54 (2) (2013) 1-11. 
[21] A. Nati, R. De Kat, F. Scarano, B. Van Oudheusden, Dynamic pitching effect on a laminar separation bubble, Exp. Fluids 56 (9) (2015) 1-17.

[22] M.R. Visbal, R.E. Gordnier, M.C. Galbraith, High-fidelity simulations of moving and flexible airfoils at low Reynolds numbers, Exp. Fluids 46 (5) (2009) 903-922.

[23] A. Uranga, P.-O. Persson, M. Drela, J. Peraire, Implicit large eddy simulation of transition to turbulence at low Reynolds numbers using a discontinuous Galerkin method, Internat. J. Numer. Methods Engrg. 87 (1-5) (2011) 232-261.

[24] L.E. Jones, R.D. Sandberg N.D. Sandham, Direct numerical simulations of forced and unforced separation bubbles on an airfoil at incidence, J. Fluid Mech. 602 (2008) 175.

[25] A. Shelton, J. Abras, B. Hathaway, M. Sanchez-Rocha, M.J. Smith, S. Menon, An investigation of the numerical prediction of static and dynamic stall, in: Annual Forum Proceedings-American Helicopter Society, Vol. 61, American Helicopter Society, Inc., 2005, p. 1826.

[26] M. Genc, Numerical simulation of flow over a thin aerofoil at a high Reynolds number using a transition model, Proc. Inst. Mech. Eng. 224 (10) (2010) 2155-2164.

[27] P. Fischer, J. Kruse, J. Mullen, H. Tufo, J. Lottes, S. Kerkemeier, Open source spectral element CFD solver, 2008. https://nek5000.mcs.anl.gov/index.php/ MainPage.

[28] P. Fischer, J. Kruse, J. Mullen, H. Tufo, J. Lottes, S. Kerkemeier, Nek5000-open source spectral element CFD solver, Argonne National Laboratory, Mathematics and Computer Science Division, Argonne, IL, 2008. See https://nek5000. mcs.anl.gov/index.php/MainPage.

[29] A. Patera, A spectral element method for fluid dynamics: laminar flow in a channel expansion, J. Comput. Phys. 54 (1984) 468-488.

[30] H.M. Tufo, P.F. Fischer, Terascale spectral element algorithms and implementations, in: Proceedings of the ACM/IEEE SC99 Conference on High Performance Networking and Computing, Portland, USA, 1999.

[31] H.M. Tufo, P.F. Fischer, Fast parallel direct solvers for coarse grid problems, J. Parallel Distrib. Comput. 61 (2) (2001) 151-177.

[32] M. Deville, P. Fischer, E. Mund, High-Order Methods for Incompressible Fluid Flow, Cambridge University Press, 2002.

[33] G. Karniadakis, S. Sherwin, Spectral/hp Element Methods for Computational Fluid Dynamics, Oxford Science Publications, 2005.
[34] M. Drela, Xfoil: An analysis and design system for low Reynolds number airfoils, in: Low Reynolds Number Aerodynamics, Springer, 1989, pp. 1-12.

[35] S. Burgmann, W. Schröder, Time-resolved two-and three-dimensional measurements of transitional separation bubbles, in: Imaging Measurement Methods for Flow Analysis, Springer, 2009, pp. 83-92.

[36] C.H.K. Williamson, Vortex dynamics in the cylinder wake, Annu. Rev. Fluid Mech. 28 (1996) 477-539.

[37] R.J. Adrian, Hairpin vortex organization in wall turbulence, Phys. Fluids 19 (4) (2007) 041301.

[38] M. Landahl, A note on an algebraic instability of inviscid parallel shear flows J. Fluid Mech. 98 (02) (1980) 243-251.

[39] D. Rodriguez, V. Theofilis, On the birth of stall cells on airfoils, Theoret. Comput. Fluid Dyn. 25 (1-4) (2011) 105-117.

[40] H. Childs, E. Brugger, B. Whitlock, J. Meredith, S. Ahern, D. Pugmire, K. Biagas M. Miller, C. Harrison, G.H. Weber, H. Krishnan, T. Fogal, A. Sanderson, C. Garth, E.W. Bethel, D. Camp, O. Rübel, M. Durant, J.M. Favre, P. Navrátil, VisIt: An enduser tool for visualizing and analyzing very large data, in: High Performance Visualization-Enabling Extreme-Scale Scientific Insight, 2012, pp. 357-372.

[41] L. Sirovich, Turbulence and the dynamics of coherent structures. Part i: Coherent structures, Quart. Appl. Math. 45 (3) (1987) 561-571.

[42] J. Lumley, Stochastic Tools in Turbulence, Dover Publications, 2007.

[43] E. Åkervik, J. Hoepffner, U. Ehrenstein, U. Henningson, Optimal growth, model reduction and control in a separated boundary layer flow using global eigenmodes, J. Fluid Mech. (2007).

[44] C.W. Rowley, Model reduction for fluids using balanced proper orthogonal decomposition, Int. J. Bifurcation Chaos 15 (2005) 997-1013.

[45] P. Schmid, J. Sesterhenn, Dynamic mode decomposition of experimental data, in: 8th International Symposium on Particle Image Velocimetry, 2008.

[46] C.W. Rowley, V. Mezić, S. Bagheri, P. Schlatter, D. Henningson, Spectral analysis of nonlinear flows, J. Fluid Mech. 641 (2009) 115-127.

[47] P. Schmid, Dynamic mode decomposition of numerical and experimental data, J. Fluid Mech. 656 (2010) 5-28.

[48] K. Chen, J.H. Tu, C.W. Rowley, Variants of dynamic mode decomposition: boundary condition, koopman, and fourier analyses, J. Nonlinear Sci. 22 (6) (2012) 887-915. 